\title{
Multiple solutions for an inhomogeneous semilinear elliptic equation in $\mathbb{R}^{N}$
}

\author{
Yinbin Deng * \\ Department of Mathematics, Huazhong Normal University \\ Wuhan, 430079 P.R.CHINA \\ $\mathrm{Yi} \mathrm{Li}{ }^{\dagger}$ and Xuejin Zhao $\ddagger$ \\ Department of Mathematics University of Iowa \\ Iowa City, IA 52242 USA
}

November 25, 1998

\section{Introduction}

In this paper, we will investigate the existence of multiple solutions for the general inhomogeneous elliptic problem

$$
\left\{\begin{array}{l}
-\triangle u+u=f(x, u)+\mu h(x), \quad x \in \mathbb{R}^{N}, \\
u \in H^{1}\left(\mathbb{R}^{N}\right),
\end{array}\right.
$$

where $h \in H^{-1}\left(\mathbb{R}^{N}\right), N \geq 2,|f(x, u)| \leq C_{1} u^{p-1}+C_{2} u$ with $C_{1}>0, C_{2} \in[0,1)$ being some constants and $2<p<+\infty$.

${ }^{*}$ Research supported in part by the Natural Science Foundation of China and NSEC

${ }^{\dagger}$ Research supported in part by the Natural Science Foundation of China and NSEC

${ }^{\ddagger}$ Research supported in part by the University of Iowa, Department of Mathematics Summer Research Fellowship 
The homogeneous case, i.e., $\mu=0$ which means 0 is a trivial solution of $(1.1)_{\mu}$, has been studied extensively (see for example [Ba], [BaLi], [BaL], [BC], [BL], [DN], [G], [KZ], [L], [L1], [L2], [Li], [N], [PW], [W]). For the nonhomogeneous case $(\mu \neq 0)$, X.P. Zhu [Z] has first studied the special case of $(1.1)_{\mu}$ with $f(x, u)=u^{p}, \mu=1$, and $1<p<\frac{N+2}{N-2}$. In some cases existence of two positive solutions was obtained if

$$
\left(\int_{\mathbb{R}^{N}} h^{2}(x) d x\right)^{\frac{1}{2}} \leq \frac{p-1}{2 p}\left(\frac{p}{2 p-2}\right)^{\frac{1}{p-2}} S^{\frac{p}{2(p-2)}}
$$

with $h(x) \geq 0, h(x) \leq C \exp (-(1+\varepsilon)|x|)$, where

$$
S=\inf \left\{\left.\int_{\mathbb{R}^{N}}\left(|\nabla u|^{2}+u^{2}\right) d x\left|u \in H^{1}\left(\mathbb{R}^{N}\right), \int_{\mathbb{R}^{N}}\right| u\right|^{p} d x=1\right\}
$$

and $C>0, \varepsilon>0$ are some constants. Y.B. Deng and Yi Li have also considered the problem $(1.1)_{\mu}$ with $f(x, u)=u^{p}$ for more general $p \in(1, \infty)$, and different assumptions on $h(x)$. Some existence and bifurcation results about the multiple solutions of $(1.1)_{\mu}$ have been obtained in [DL1], [DL2] if $|x|^{N-2} h(x)$ is bounded in $\mathbb{R}^{N}$. X.P. Zhu and H.S. Zhou [ZZ] have considered a more general inhomogeneous problem:

$$
\begin{cases}-\triangle u+u=\lambda(f(u)+h(x)), & x \in \mathbb{R}^{N}, \\ u \in H^{1}\left(\mathbb{R}^{N}\right), & N \geq 2,\end{cases}
$$

with $h(x) \in L^{2}\left(\mathbb{R}^{N}\right) \cap L^{\beta}\left(\mathbb{R}^{N}\right)$ for some $\beta>\frac{N}{2}$. They have shown that there exists a $\lambda^{*}>0$ such that problem (1.3) admits at least two positive solutions if $\lambda \in\left(0, \lambda^{*}\right)$ and no positive solution if $\lambda>\lambda^{*}$ under some assumptions on $f$. However, their method cannot give an estimate of $\lambda^{*}$. A similar problem,

$$
\left\{\begin{array}{l}
-\triangle u+u=\lambda f(u+h), \quad x \in \Omega, \\
\left.u\right|_{\Omega}=0, u>0, u \in H_{0}^{1}(\Omega),
\end{array}\right.
$$

with $\Omega$ an exterior domain, has been discussed by J.F. Yang $[\mathrm{Y}]$ if $h(x) \in C^{\alpha}(\Omega) \cap L^{2}(\Omega)$.

D.M. Cao and H.S. Zhu [CZ] have studied problem $(1.1)_{\mu}$ with $\mu=1, h \in H^{-1}\left(\mathbb{R}^{N}\right)$ recently. They have proved, under some conditions on $f(x, u)$, that problem $(1.1)_{\mu}$ possesses at least two solutions if $\|h\|_{H^{-1}\left(\mathbb{R}^{N}\right)}<C_{p} S^{\frac{p}{2(p-2)}}$ and no solutions if $f(x, u)=u^{p}$ 
$\left(1<p<\frac{N+2}{N-2}\right)$ and $\|h\|_{H^{-1}\left(\mathbb{R}^{N}\right)}$ is large enough. There is no information about the multiple results (the existence of three solutions) and bifurcation results in their paper. On the other hand, they need the growth of the nonlinear function $f(x, u)$ to be lower than the critical exponent.

In this paper, stimulated by [CZ, DL2] we will consider the existence of multiple solutions for problem $(1.1)_{\mu}$ with $h(x) \in L^{\infty}\left(\mathbb{R}^{N}\right) \cap H^{-1}\left(\mathbb{R}^{N}\right)$ and $\mu>0$. More precisely, we assume $f(x, t)$ satisfies the following basic conditions throughout this paper:

$\left.\mathrm{f}_{1}\right) f(x, u) \in C^{1}\left((0, \infty), \mathbb{R}^{1}\right)$ with respect to $u$;

$\left.\mathrm{f}_{2}\right)$ there exist $C_{1}>0, C_{2} \in[0,1)$ such that $|f(x, t)| \leq C_{1}|t|^{p-1}+C_{2} t$ for $x \in \mathbb{R}^{N}$, $t \in(-\infty, \infty)$ and $\lim _{t \rightarrow \infty} \frac{f(x, t)}{t}=+\infty$ uniformly for $x \in \mathbb{R}^{N}$ where $2<p<+\infty$ and $N \geq 2$;

$\left.\mathrm{f}_{3}\right)$ there exists a constant $\alpha \in(0,1)$ such that $\alpha t f_{t}^{\prime}(x, t) \geq f(x, t) \geq 0$ for all $x \in \mathbb{R}^{N}$ $t \in(0, \infty)$.

and

h) $h(x) \in L^{\infty}\left(\mathbb{R}^{N}\right) \cap H^{-1}\left(\mathbb{R}^{N}\right), \quad h(x) \geq 0, \quad h(x) \not \equiv 0$ in $\mathbb{R}^{N}$ and $\lim _{|x| \rightarrow \infty} h(x)=0$

Our main results are as follows.

Theorem 1.1. If $\left.\mathrm{f}_{1}\right)-\mathrm{f}_{3}$ ) and $h$ ) hold, there exists a positive constant $\mu^{*}<+\infty$ such that problem $(1.1)_{\mu}$ has at least one minimal positive solution $u_{\mu}$ if $\mu \in\left(0, \mu^{*}\right)$ and there are no solutions for $(1.1)_{\mu}$ if $\mu>\mu^{*}$ and $u_{\mu}$ is increasing with respect to $\mu \in\left(0, \mu^{*}\right]$; furthermore, there is a unique solution for (1.1) $\mu_{\mu^{*}}$ if $p \leq \frac{2 N}{N-2}$ when $N \geq 3$.

Defining the variational functional of $(1.1)_{\mu}$ by

$$
I(u)=\frac{1}{2} \int_{\mathbb{R}^{N}}\left(|\nabla u|^{2}+u^{2}\right) d x-\int_{\mathbb{R}^{N}} F(x, u) d x-\mu \int_{\mathbb{R}^{N}} h(x) u d x,
$$

where $F(x, u)=\int_{0}^{u} f(x, t) d t$, we have the next theorem.

Theorem 1.2. If in addition to $\left.\mathrm{f}_{1}\right), \mathrm{f}_{3}$ ), and $\mathrm{f}_{2}$ ) with $p<\frac{2 N}{N-2}$ if $N \geq 3$ and h) we also have 
$\left.\mathrm{f}_{4}\right) f(x, \cdot) \in C^{2}(0,+\infty), \frac{\partial^{2} f}{\partial t^{2}} \geq 0$ for $x \in \mathbb{R}^{N}, t \geq 0$,

$\mathrm{f}_{5}$ ) $\lim _{t \rightarrow 0^{+}} t \cdot \frac{\partial^{2} f}{\partial t^{2}}=0$ uniformly for $x \in \mathbb{R}^{N}, t \geq 0, \lim _{t \rightarrow \infty} t^{1-q}\left|\frac{\partial^{2} f}{\partial t^{2}}\right| \leq C$ uniformly for $x \in \mathbb{R}^{N}$ where $C>0$ is some constant and $0<q<\frac{4}{N-2}$,

$\left.\mathrm{f}_{6}\right) \lim _{|x| \rightarrow \infty} f(x, t)=\bar{f}(t)$ uniformly for bounded $t>0$ and $f(x, t) \geq \bar{f}(t)$ for all $x \in \mathbb{R}^{N}$,

then problem $(1.1)_{\mu}$ has at least two positive solutions $u_{\mu}, U_{\mu}$ with $u_{\mu}<U_{\mu}$ if $\mu \in\left(0, \mu^{*}\right)$ and $u_{\mu}$ is a local minimizer of $I(u)$.

Remark 1.3. The assumption that $p<\frac{2 N}{N-2}$ if $N \geq 3$ in this general setting is optimal in the sense that if $p=\frac{2 N}{N-2}$ then in [DL1] Y.B. Deng and Yi Li proved that for $f=u^{p-1}$ and $h$ under some monotonic property the problem $(1.1)_{\mu}$ has only one solution (namely the minimal solution) for $\mu$ small.

It is well known that due to the fact that the Sobolev embedding

$$
H^{1}\left(\mathbb{R}^{N}\right) \hookrightarrow L^{q}\left(\mathbb{R}^{N}\right) \quad\left(2 \leq q \leq \frac{2 N}{N-2}\right)
$$

is not compact, the variational functional $I(u)$ fails to satisfy the so-called PS (PalaisSmale) condition. Such a failure causes difficulty in applying the variational approach to $(1.1)_{\mu}$. Furthermore, when $\mu \nRightarrow 0,0$ is no longer a trivial solution of $(1.1)_{\mu}$, and therefore the mountain pass lemma cannot be applied directly; since the nonlinearity $f(x, u)$ is very general, we do not even need any growth assumption on $f(x, u)$ when we get the first solution, so the methods in [CZ] cannot be used. It should be noted that the method of getting the second solution in [DL2] depends strongly on the case $f(x, u)=u^{p}$, so the method in [DL2] is also not usable. To overcome the difficulties mentioned above, we first consider the special case. The problem

$$
\begin{cases}-\triangle u+u=u^{p}+\mu h(x), & \text { in } \mathbb{R}^{N}, \\ u \in H^{1}\left(\mathbb{R}^{N}\right), & u>0 \text { in } \mathbb{R}^{N}\end{cases}
$$

possesses at least one solution if $\mu \in\left(0, \mu_{1}\right)$ for some positive constant $\mu_{1}>0$ by the sup-sub solution method. Then by applying the comparison principle we get the minimal 
solution for problem (1.1) $)_{\mu}$ if $\mu \in\left(0, \mu^{*}\right]$. If $\mu \in\left(0, \mu^{*}\right)$, we show that a minimal positive solution of $(1.1)_{\mu}$ is also a local minimizer of $I(u)$ and we obtain a sequence of approximated solutions by application of the mountain pass lemma. We establish the existence of a second solution by using concentration-compactness arguments to obtain a precise understanding of the lack of compactness for the sequence of approximated solutions.

Remark 1.4. In this paper, we are only concerned with the positive solution of $(1.1)_{\mu}$, so we always suppose $f(x, u) \equiv 0$ for all $x \in \mathbb{R}^{N}$ and $u<0$

\section{The minimal solution for a special problem}

In this section, we discuss the existence of the minimal solution of a special problem:

$$
\left\{\begin{array}{l}
-\triangle u+u=u^{p-1}+\nu h(x) \\
u \in L^{\infty}\left(\mathbb{R}^{N}\right), \quad u>0 \text { in, } \mathbb{R}^{N} .
\end{array}\right.
$$

by using a standard barrier method. Since $h$ has no explicit decay property, a more careful choice of supersolution is needed here. We will use the Green's function of $-\Delta+1$ on $R^{N}$ which was given by

$$
0<G(|x-y|)=\left(|x-y|^{-\frac{N-2}{2}} K_{\frac{N-2}{2}}(|x-y|),\right.
$$

where

$$
K_{\gamma}(z)=\frac{1}{\Gamma\left(\gamma+\frac{1}{2}\right)}\left(\frac{\pi}{2}\right)^{\frac{1}{2}} \frac{e^{-z}}{z^{\frac{1}{2}}} \int_{0}^{\infty} e^{-t} t^{\gamma-\frac{1}{2}}\left(1+\frac{t}{2 z}\right)^{\gamma-\frac{1}{2}} d t
$$

denotes the modified Bessel function of order $\gamma$. In the Appendix $\mathrm{C}$ of [GNN], Gidas, $\mathrm{Ni}$ and Nirenberg summarized the properties of $K_{\gamma}$. In particular, $G$ satisfies:

$$
\begin{gathered}
G(r) \leq C \frac{e^{-r}}{r^{N-2}}(1+r)^{\frac{N-3}{2}}, \\
\frac{G_{r}^{\prime}(r)}{G(r)} \longrightarrow-1 \quad \text { as } r \longrightarrow \infty .
\end{gathered}
$$

For $N=3$

$$
G(r)=\left(\frac{\pi}{2}\right)^{\frac{1}{2}}\left(\frac{e^{-r}}{r}\right) .
$$


By some standard argument we can get the Lemma.

Lemma 2.1. Suppose $h \in L^{\infty}\left(\mathbb{R}^{N}\right)$. Then

$$
w(x)=\int_{\mathbb{R}^{N}} G(|x-y|) h(y) d y
$$

is a solution of the linear problem

$$
-\triangle w+w=h(x) \text { in } R^{N}
$$

Furthermore, if $h(y) \longrightarrow 0$ as $|y| \longrightarrow \infty$, then $w(x) \longrightarrow 0$ as $|x| \longrightarrow \infty$.

Lemma 2.2 . Assume h), The problem $(2.1)_{\nu}$ possesses a minimal solution for all $\nu \in\left(0, \nu_{1}\right)$. Where

$$
\nu_{1}=\frac{1-2^{2-p}}{2 \int_{\mathbb{R}^{N}} G\left(\left|\left(1-2^{2-p}\right)^{\frac{1}{2}} x-y\right|\right) h\left(\frac{y}{\left(1-2^{2-p}\right)^{\frac{1}{2}}}\right) d y} .
$$

\section{Proof.}

Let

$$
\tilde{u}=\frac{\nu}{1-2^{2-p}} \int_{\mathbb{R}^{N}} G\left(\left|\left(1-2^{2-p}\right)^{\frac{1}{2}} x-y\right|\right) f\left(\frac{y}{\left(1-2^{2-p}\right)^{\frac{1}{2}}}\right) d y .
$$

Then for $h(x) \in L^{\infty}\left(R^{N}\right)$ we have, by (2.3),

$$
\begin{array}{rl}
\int_{\mathbb{R}^{N}} & G(|x-y|) h\left(\frac{y}{\left(1-2^{2-p}\right)^{\frac{1}{2}}}\right) d y \\
& \leq \int_{\{|x-y| \leq a\}} G(|x-y|) h\left(\frac{y}{\left(1-2^{2-p}\right)^{\frac{1}{2}}}\right) d y \\
& +\int_{\{|x-y| \geq a\}} G(|x-y|) h\left(\frac{y}{\left(1-2^{2-p}\right)^{\frac{1}{2}}}\right) d y \\
& \leq C \int_{0}^{a} r d r+C \int_{a}^{\infty} \frac{e^{-r}}{r^{\frac{N-1}{2}}} r^{N-1} d r \\
& \leq C_{1}
\end{array}
$$

So we have

$$
\begin{aligned}
\tilde{u}(x) & =w\left(\left(1-2^{2-p}\right)^{\frac{1}{2}} x\right) \\
& =\frac{\nu}{1-2^{2-p}} \int_{\mathbb{R}^{N}} G\left(\left|\left(1-2^{2-p}\right)^{\frac{1}{2}} x-y\right|\right) h\left(\frac{y}{\left(1-2^{2-p}\right)^{\frac{1}{2}}}\right) d y \\
& \leq \frac{1}{2}
\end{aligned}
$$

if we choose

$$
\nu \leq \nu_{1}=\frac{1-2^{2-p}}{2 \int_{\mathbb{R}^{N}} G\left(\left|\left(1-2^{2-p}\right)^{\frac{1}{2}} x-y\right|\right) h\left(\frac{y}{\left(1-2^{2-p}\right)^{\frac{1}{2}}}\right) d y} .
$$


Now we check that $\tilde{u}$ must be a supersolution of $(2.1)_{\nu}$ if $\nu \leq \nu_{1}$. In fact, from Lemma 2.1

$$
w(x)=\frac{\nu}{\alpha^{2}} \int_{\mathbb{R}^{N}} G(|x-y|) h\left(\frac{y}{\alpha}\right) d y
$$

must be the solution of

$$
-\triangle w+w=\frac{\nu}{\alpha^{2}} h\left(\frac{x}{\alpha}\right) \text { in } R^{N} .
$$

Then $\tilde{u}=w(\alpha x)$ must be the solution of

$$
-\triangle \tilde{u}+\alpha^{2} \tilde{u}=\nu h(x) \text { in } R^{N}
$$

Taking $\alpha=\left(1-2^{2-p}\right)^{\frac{1}{2}}$, by $(2.8)$ we have

$$
\begin{aligned}
- & \triangle \tilde{u}+\tilde{u}-\tilde{u}^{p-1}-\nu h(x) \\
& =\triangle \tilde{u}+\tilde{u}\left(1-\tilde{u}^{p-2}\right)-\nu h(x) \\
& \geq \triangle \tilde{u}+\tilde{u}\left(1-\left(\frac{1}{2}\right)^{p-2}\right)-\nu h(x) \\
& =0
\end{aligned}
$$

if $\nu \leq \nu_{1}$. Thus $\tilde{u}$ is a supersolution of $(2.1)_{\nu}$.

On the other hand, $\underset{\sim}{u}=0$ is clearly a subsolution of $(2.1)_{\nu}$ for all $\nu>0$ and $\underset{\sim}{u}<\tilde{u}$. By the standard barrier method (see [A] Theorem 9.4 or [GE]) there exists a solution $u_{\nu}$ of $(2.1)_{\nu}$ such that $0 \leq u_{\nu} \leq \tilde{u}$. Since 0 is not a solution of $(2.1)_{\nu}$ and $h(x) \geq 0$, the maximum principle implies that $0<u_{\nu} \leq \tilde{u}$. Again using a result of Amann (see [A] Theorem 9.4 or $[\mathrm{GE}]$ ) we can choose a minimal solution $u_{\nu}$ in the order interval $[0, \tilde{u}]$ by an iteration scheme with initial value $u_{(0)}=\underset{\sim}{u} \equiv 0$.

In the following, we show that $u_{\nu}$ is minimal among all solutions of $(2.1)_{\nu}$. In fact, let $u$ be any other solution of $(2.1)_{\nu}$, then $\tilde{u}^{*}=u$ may be considered as a supersolution of $(2.1)_{\nu}$. Clearly, $\underset{\sim}{u^{*}}=0$ is a subsolution of $(2.1)_{\nu}$. By using the result of Amann we can obtain a minimal solution $u_{\nu}^{*}$ in the order interval $[0, \mathrm{u}]$ by an iteration scheme with initial value $u_{(0)}=\underset{\sim}{u^{*}}=0$. Because $\underset{\sim}{u^{*}}=\underset{\sim}{u}=0$ we deduce that $u_{\nu}^{*} \equiv u_{\nu}$. Thus

$$
0=\underset{\sim}{u}<u_{\nu} \equiv u_{\nu}^{*} \leq \tilde{u}^{*} \equiv u .
$$

Since

$$
0<u_{\nu} \leq \tilde{u} \leq \frac{1}{2}
$$


we have $u_{\nu} \in L^{\infty}\left(R^{N}\right)$.

Remark 2.3. From the proof of Lemma 2.2 we conclude that there exists a constant $\bar{a}>0$ such that

$$
1-(p-1) u_{\nu}^{p-2} \geq \bar{a} \quad \text { for } \quad \nu \in\left(0, \nu_{1}\right)
$$

Lemma 2.4. The problem $(2.1)_{\nu}$ has no solution if $\nu>\nu_{3}$, where $\nu_{3}$ is given by

$$
\nu_{3}=\frac{\int_{0}^{\frac{1}{2}(1+2 N)^{\frac{1}{2}}} \phi(r)\left(1+2 N-4 r^{2}\right)^{\frac{p-1}{p-2}} r^{N-1} d r}{\int_{\mathbb{R}^{N}} \phi h d x}
$$

where $\phi=e^{-r^{2}}$.

Proof. Let $u_{\nu}$ is a positive solution of $(2.1)_{\nu}$. Then

$$
\begin{gathered}
-\triangle u_{\nu}+u_{\nu}=u_{\nu}^{p-1}+\nu h(x), \quad x \in \mathbb{R}^{N}, \quad N>2 \\
u_{\nu} \in L^{\infty}\left(\mathbb{R}^{N}\right), \quad u>0 \quad \text { in } \mathbb{R}^{N},
\end{gathered}
$$

Taking $\phi \in C^{2}\left(R^{N}\right), \phi>0$ in $R^{N}, \phi(x) \longrightarrow 0$ as $|x| \longrightarrow \infty$ and the decay must be fast enough at $\infty$ so that

$$
\int_{\mathbb{R}^{N}} \phi \triangle u_{\nu} d x=\int_{\mathbb{R}^{N}} \triangle \phi u_{\nu} d x .
$$

With this fact we can deduce that

$$
\int_{\mathbb{R}^{N}}\left(\triangle \phi u_{\nu}-\phi u_{\nu}+\phi u_{\nu}^{p-1}+\nu \phi h\right) d x=0
$$

So we have

$$
\nu=\frac{\int_{\mathbb{R}^{N}}\left(\phi\left(u_{\nu}-u_{\nu}^{p-1}\right)-\triangle \phi u_{\nu}\right) d x}{\int_{\mathbb{R}^{N}} \phi h d x}
$$

Fix $\phi(x)=\phi(r)=e^{-r^{2}}$, then

$$
\phi^{\prime}(r)=-2 r e^{-r^{2}}, \quad \phi^{\prime \prime}(r)=e^{-r^{2}}\left(-2+4 r^{2}\right) .
$$

Thus

$$
\begin{aligned}
\triangle \phi & =\phi^{\prime \prime}(r)+\frac{N-1}{r} \phi^{\prime} \\
& =e^{-r^{2}}\left(-2+4 r^{2}\right)-\frac{N-1}{r} 2 r e^{-r^{2}} \\
& =\left[4 r^{2}-2 N\right] \phi .
\end{aligned}
$$


So

$$
\nu=\frac{\int_{\mathbb{R}^{N}}\left(\phi u_{\nu}\left(1+2 N-4 r^{2}-u_{\nu}^{p-2}\right)\right) d x}{\int_{\mathbb{R}^{N}} \phi h d x}
$$

where $r=|x|$. Set

$$
K\left(r, u_{\nu}\right)=1+2 N-4 r^{2}-u_{\nu}^{p-2}
$$

we have

$$
\begin{aligned}
\nu & =\frac{\int_{\mathbb{R} N}\left(\phi u_{\nu} K\left(r, u_{\nu}\right)\right) d x}{\int_{\mathbb{R}^{N}} \phi h d x} \\
& =\frac{\int_{K\left(|x|, \nu_{\nu}\right) \geq 0}\left(\phi u_{\nu} K\left(r, u_{\nu}\right)\right) d x}{\int_{\mathbb{R}^{N}} \phi h d x}+\frac{\int_{K\left(|x|, u_{\nu}\right) \leq 0}\left(\phi u_{\nu} K\left(r, u_{\nu}\right)\right) d x}{\int_{\mathbb{R}^{N}} \phi h d x} \\
& \leq \frac{\int_{K\left(|x|, u_{\nu}\right) \geq 0}\left(\phi u_{\nu} K\left(r, u_{\nu}\right)\right) d x}{\int_{\mathbb{R}^{N}} \phi h d x}
\end{aligned}
$$

From $K\left(|x|, u_{\nu}\right) \geq 0$ we have

$$
0<u_{\nu} \leq\left(1+2 N-4 r^{2}\right)^{\frac{1}{p-2}} \text { and } r \leq \frac{1}{2}(1+2 N)^{\frac{1}{2}} .
$$

By the definition of $K\left(r, u_{\nu}\right)$ we have

$$
\nu \leq \frac{\int_{0}^{\frac{1}{2}(1+2 N)^{\frac{1}{2}}} \phi\left(1+2 N-4 r^{2}\right)^{\frac{p-1}{p-2}} r^{N-1} d r}{\int_{\mathbb{R}^{N}} \phi h d x}=\nu_{3} \leq \infty .
$$

Lemma 2.5. (Lax-Milgram Lemma) (see [E]). Assume that

$$
B: H \times H \longrightarrow R^{N}
$$

is the bilinear mapping for which that exists constants $\alpha, \beta>0$ such that

$$
|B[u, v]| \leq \alpha\|u\|\|v\|, \text { for all } u, v \in H
$$

and

(ii) $\quad \beta\|u\|^{2} \leq B[u, u]$ for all $u \in H$.

Finally, let $h: H \longrightarrow R$ be a bounded linear functional on $H$. Then there exists a unique element $u \in H$, such that

$$
B[u, v]=<h, v>, \text { for all } v \in H .
$$

Lemma 2.6. If h) holds, then $u_{\nu}(x) \in L^{\infty}\left(R^{N}\right) \cap H^{1}\left(\mathbb{R}^{N}\right)$ if $\nu \in\left(0, \nu_{1}\right)$.

Proof. Because $u_{\nu}$ is a solution of $(2.1)_{\nu}$, then

$$
-\triangle u_{\nu}+u_{\nu}\left(1-u_{\nu}^{p-2}\right)-\nu h=0 .
$$


Let $a(x)=1-u_{\nu}^{p-2}$. Because $\tilde{u}$ is a supersolution and $u_{\nu}$ is a minimal solution of $(2.1)_{\nu}$, we have by Remark 2.3 , that

$$
a(x)=1-u_{\nu}^{p-2} \geq 1-\tilde{u}^{p-2} \geq \bar{a}
$$

if $\nu>0$ small enough. Define

$$
B[u, v]=\int_{\mathbb{R}^{N}} \nabla u \nabla v d x+\int_{\mathbb{R}^{N}} a(x) u v d x \quad u, \quad v \in H^{1}\left(\mathbb{R}^{N}\right) .
$$

Then by Holder inequality,

$$
\|B[u, v]\| \leq C\|u\|\|v\|
$$

and

$$
\begin{aligned}
B[u, u] & =\int_{\mathbb{R}^{N}}|\nabla u|^{2} d x+\int_{\mathbb{R}^{N}} a(x) u^{2} d x \\
& \geq \int_{\mathbb{R}^{N}}|\nabla u|^{2} d x+\int_{\mathbb{R}^{N}} \bar{a} u^{2} d x \\
& \geq C\|u\|^{2}
\end{aligned}
$$

for all $u \in H^{1}\left(\mathbb{R}^{N}\right)$. From $h \in H^{-1}\left(R^{N}\right) \cap L^{\infty}\left(R^{N}\right)$ and Lemma 2.5 we deduce that

$$
B[u, v]=<h, v>\text { for all } v \in H^{1}\left(\mathbb{R}^{N}\right)
$$

has a unique solution. That means that $u$ is a weak solution of

$$
-\triangle u+a(x) u=\nu h, \quad u \in H^{1}\left(\mathbb{R}^{N}\right) .
$$

Because $u_{\nu} \in L^{\infty}\left(R^{N}\right)$ and $h \in L^{\infty}\left(R^{N}\right)$ we can easily deduce that $u$ is also a classical solution of

$$
\left\{\begin{array}{l}
-\triangle u+a(x) u=\nu h \\
u(x) \longrightarrow 0 \text { as }|x| \longrightarrow \infty
\end{array}\right.
$$

On the other hand, if $h(x) \longrightarrow 0$ as $|x| \longrightarrow \infty$, by Lemma 2.1, we can deduce $u_{\nu}$ is also a solution of (2.13) . By the uniqueness of the solution of (2.13) we have $u \equiv u_{\nu}$. Thus $u_{\nu} \in H^{1}\left(\mathbb{R}^{N}\right)$.

Theorem 2.7. Suppose h) and $p>2$. Then there exists a positive constant $\nu^{*}<+\infty$ such that problem

$$
\begin{cases}-\triangle u+u=u^{p-1}+\nu h(x), & \\ u \in H^{1}\left(\mathbb{R}^{N}\right), & u>0 \text { in } \mathbb{R}^{N},\end{cases}
$$


possesses a minimal solution if $\nu \in\left(0, \nu^{*}\right)$ and there is no solution for problem $(2.1)_{\nu}$ if $\nu>\nu^{*}$. Furthermore, if $h(x) \rightarrow 0$ as $|x| \rightarrow \infty$, and $p<\frac{2 N}{N-2}$ when $N \geq 3$, then problem $(2.1)_{\nu}$ possesses only one solution if $\nu=\nu^{*}$.

Proof. From Lemma 2.4 we set

$$
\nu^{*}=\sup \left\{\nu>0 \mid(2.14)_{\nu} \text { possesses at least one solution in } H^{1}\left(\mathbb{R}^{N}\right)\right\}
$$

By Lemma 2.2 and Lemma 2.4 we have

$$
0<\nu_{1} \leq \nu^{*}<\nu_{2}<+\infty
$$

For any $\nu \in\left(0, \nu^{*}\right)$, by the definition of $\nu^{*}$ we can find a $\bar{\nu} \in\left(\nu, \nu^{*}\right)$ such that $(2.14)_{\bar{\nu}}$ have a solution $u_{\bar{\nu}}$ and

$$
-\triangle u_{\bar{\nu}}+u_{\bar{\nu}}-u_{\bar{\nu}}^{p-1}-\nu h(x)=(\bar{\nu}-\nu) h(x) \geq 0 .
$$

Thus $\tilde{u}=u_{\bar{\nu}}$ is a supersolution of $(2.14)_{\nu}$. From $h(x) \geq 0$ we deduce that $u \equiv 0$ is a subsolution of $(2.14)_{\nu}$ for all $\nu>0$. Again by the standard barrier method as in the proof of Lemma 2.2 , we can obtain a solution $u_{\nu}$ of $(2.14)_{\nu}$ such that $0 \leq u_{\nu} \leq u_{\bar{\nu}}$. Since $u_{\nu}$ can be derived by an iteration scheme with initial value $u_{(0)}=0, u_{\nu}$ is a minimal solution of $(2.14)_{\nu}$. Since 0 is not a solution of $(2.14)_{\nu}, \bar{\nu}>\nu$ and $h(x) \geq 0$, the maximum principle implies that

$$
0<u_{\nu}<u_{\bar{\nu}}
$$

Furthermore

$$
\begin{aligned}
& \int_{\mathbb{R}^{N}}\left(\left|\nabla u_{\nu}\right|^{2}+u_{\nu}^{2}\right) d x=\int_{\mathbb{R}^{N}} u_{\nu}^{p} d x+\nu \int_{\mathbb{R}^{N}} h(x) u_{\nu} d x \\
& \leq \int_{\mathbb{R}^{N}} u_{\bar{\nu}}^{p} d x+\bar{\nu} \int_{\mathbb{R}^{N}} h(x) u_{\bar{\nu}} d x \\
& =\int_{\mathbb{R}^{N}}\left(\left|\nabla u_{\bar{\nu}}\right|^{2}+u_{\bar{\nu}}^{2}\right) d x<+\infty .
\end{aligned}
$$

Thus $u_{\nu} \in H^{1}\left(\mathbb{R}^{N}\right)$.

By the definition of $\nu^{*}$ we can conclude that $(2.14)_{\nu}$ have no solution in $H^{1}\left(\mathbb{R}^{N}\right)$ for $\nu>\nu^{*}$.

The existence and the uniqueness of $(2.14)_{\nu^{*}}$ can be proved in the same way as those in [DL2] if we assume that $p \in\left(2, \frac{2 N}{N-2}\right]$ if $N \geq 3$.

Corollary 2.8. Let $h \in L^{\infty}\left(\mathbb{R}^{N}\right)$. Then there exists a constant $\nu^{*}>0$ such that 
i) $(2.14)_{\nu}$ possesses a minimal solution for all $\nu \in\left(0, \nu^{*}\right)$ and $p>2$ and $u_{\nu}$ is increasing with respect to $\nu$;

ii) there are no solutions of $(2.14)_{\nu}$ for $\nu>\nu^{*}$.

Unfortunately, we couldn't get the existence of the solution for $(2.14)_{\nu}$ when $\nu=\nu^{*}$.

\section{The existence of the minimal solution}

In this section, we first consider the problem

$$
\begin{cases}-\triangle u+u=C_{1} u^{p-1}+C_{2} u+\mu h(x), & x \in \mathbb{R}^{N}, \\ u \in H^{1}\left(\mathbb{R}^{N}\right), & u>0 \text { in } \mathbb{R}^{N},\end{cases}
$$

where, as before, $C_{1}>0, C_{2} \in[0,1)$ are some constants which are given by $\left.\mathrm{f}_{2}\right), p>2$. We will first prove that there exists a constant $\mu^{*}>0$ and $\mu^{*}<\infty$, such that problem (3.1) $\mu$ has a minimal solution if $\mu \in\left(0, \mu^{*}\right]$ and no solution for (3.1) if $\mu>\mu^{*}$.

Using the theorem 2.7, we can get the following corollary.

Corollary 3.1. Suppose h) holds. Then there exists a positive constant $\bar{\mu}^{*}<+\infty$ such that problem $(3.1)_{\mu}$ possesses a minimal solution if $\mu \in\left(0, \bar{\mu}^{*}\right)$ and there is no solution for $(3.1)_{\mu}$ if $\mu>\bar{\mu}^{*}$. For $p \leq \frac{2 N}{N-2}$ when $N \geq 3$, then problem $(3.1)_{\mu}$ possesses only one solution if $\mu=\mu^{*}$

Proof. Suppose $u(x)$ to be a solution of $(2.14)_{\nu}$. Denote $w(x)=M u(\alpha x)$ with positive $M, \alpha$ be determined later. Then

$$
\begin{aligned}
0 & =-\triangle u+u-u^{p-1}-\nu h(x) \\
& =-\frac{1}{M \alpha^{2}} \triangle w+\frac{1}{M} w-\frac{1}{M^{p-1}} w^{p-1}-\nu h(x) .
\end{aligned}
$$

Thus $-\triangle w+\alpha^{2} w-\frac{\alpha^{2}}{M^{p-2}} w^{p-1}-\nu \alpha^{2} M h(x)=0$. Taking $\alpha=\left(1-C_{2}\right)^{\frac{1}{2}}, M=\left(\frac{1-C_{2}}{C_{1}}\right)^{\frac{1}{p-2}}$, we have

$$
-\triangle w+\left(1-C_{2}\right) w=C_{1} w^{p-1}+\nu\left(1-C_{2}\right)\left(\frac{1-C_{2}}{C_{1}}\right)^{\frac{1}{p-2}} h(x) .
$$


Denote $\bar{\mu}^{*}=\nu^{*}\left(1-C_{2}\right)\left(\frac{1-C_{2}}{C_{1}}\right)^{\frac{1}{p-2}}>0$. We deduce that $w(x)$ is a solution of $(3.1)_{\mu}$ if $0<\mu \leq \bar{\mu}^{*}$. Then the conclusion follows from Theorem 2.7 and Corollary 2.8.

Now we are ready to prove the existence of the minimal solutions.

Lemma 3.2. Suppose $\left.\mathrm{f}_{1}\right), \mathrm{f}_{2}$ ) and $h$ ). Then there exists a positive constant $\mu^{*}<+\infty$ such that problem $(1.1)_{\mu}$ possesses a minimal solution for $\mu \in\left(0, \mu^{*}\right)$.

Proof. From Corollary 2.1, problem $(3.1)_{\mu}$ has a minimal solution $\tilde{u}_{\mu}$ if $\mu \in\left(0, \bar{\mu}^{*}\right)$. By $\left.\mathrm{f}_{2}\right)$ $\tilde{u}_{\mu}$ must be a supersolution of $(1.1)_{\mu}$. On the other hand, $\underset{\sim}{u}=0$ is a subsolution of $(1.1)_{\mu}$. Thus by the standard barrier method, $(1.1)_{\mu}$ has a solution $u_{\mu}$ such that $0 \leq u_{\mu} \leq \tilde{u}_{\mu}$. (See [GE].) Since $h(x) \geq 0$ and $h(x) \nRightarrow 0$ we conclude that $u_{\mu}>0$ in $\mathbb{R}^{N}$ by the maximum principle. Set

$$
\mu^{*}=\sup \left\{\mu>0 \mid(1.1)_{\mu} \text { possesses at least one solution }\right\} .
$$

We have $\mu^{*} \geq \bar{\mu}^{*}>0$. For any $\mu \in\left(0, \mu^{*}\right)$, by (3.2) we can find a $\bar{\mu} \in\left(\mu, \mu^{*}\right)$ such that $(1.1)_{\bar{\mu}}$ has a solution $u_{\bar{\mu}}$ and

$$
-\triangle u_{\bar{\mu}}+u_{\bar{\mu}}-f\left(x, u_{\bar{\mu}}\right)-\mu h(x)=(\bar{\mu}-\mu) h(x) \geq 0 .
$$

Thus $\tilde{u}=u_{\bar{\mu}}$ is a supersolution of $(1.1)_{\mu}$. From $h(x) \geq 0, h(x) \triangleq 0$ and $f(x, 0) \equiv 0$ we deduce $\underset{\sim}{u} \equiv 0$ is a subsolution of $(1.1)_{\mu}$ for all $\mu>0$. Again by the standard barrier method as in the proof of Lemma 2.2, we can obtain a solution $u_{\mu}$ of $(1.1)_{\mu}$ such that $0 \leq u_{\mu} \leq u_{\bar{\mu}}$. Since $u_{\mu}$ can be derived by an iteration scheme with initial value $u_{(0)}=0$, $u_{\mu}$ is a minimal solution of $(1.1)_{\mu}, \bar{\mu}>\mu$, and $h(x) \geq 0, h(x) \triangleq 0$, the maximum principle implies that

$$
0<u_{\mu}<u_{\bar{\mu}} \quad \text { if } \bar{\mu}>\mu \text {. }
$$

Furthermore,

$$
\begin{aligned}
\int_{\mathbb{R}^{N}}\left|\nabla u_{\mu}\right|^{2}+u_{\mu}^{2} d x & =\int_{\mathbb{R}^{N}} f\left(x, u_{\mu}\right) u_{\mu} d x+\mu \int_{\mathbb{R}^{N}} h(x) u_{\mu} d x \\
& \leq \int_{\mathbb{R}^{N}} f\left(x, u_{\bar{\mu}}\right) u_{\bar{\mu}} d x+\bar{\mu} \int_{\mathbb{R}^{N}} h(x) u_{\bar{\mu}} d x \\
& =\int_{\mathbb{R}^{N}}\left|\nabla u_{\bar{\mu}}\right|^{2}+\left|u_{\bar{\mu}}\right|^{2} d x<+\infty .
\end{aligned}
$$


Lemma 3.3. If $\left.\mathrm{f}_{1}\right)-\mathrm{f}_{5}$ ) hold with $p<\frac{2 N}{N-2}$ if $N \geq 3$, then the minimization problem

$$
\begin{aligned}
\lambda_{1}=\inf \left\{\int_{\mathbb{R}^{N}}|\nabla v|^{2}+\left(1-f_{u}^{\prime}(x, 0)\right) v^{2} \mid\right. & v \in H^{1}\left(\mathbb{R}^{N}\right), \\
& \left.\int_{\mathbb{R}^{N}}\left(f_{u}^{\prime}\left(x, u_{\mu}\right)-f_{u}^{\prime}(x, 0)\right) v^{2} d x=1\right\}
\end{aligned}
$$

can be achieved by some $v_{0}>0$, and furthermore, $\lambda_{1}>1$, where $u_{\mu}$ is the minimal solution of $(1.1)_{\mu}$ with $\mu \in\left(0, \mu^{*}\right)$.

Proof. Notice that $f_{u}^{\prime}(x, 0) \leq C_{2} \in[0,1), \int_{\mathbb{R}^{N}}|\nabla v|^{2}+\left(1-f_{u}^{\prime}(x, 0)\right) v^{2} \geq\left(1-C_{2}\right)\|v\|_{H^{1}\left(\mathbb{R}^{N}\right)}^{2}$. It is easy to see that $\lambda_{1}<+\infty$. Let $\left\{v_{n}\right\} \subset H^{1}\left(\mathbb{R}^{N}\right)$ be a minimizing sequence of $\lambda_{1}$, that is,

$$
\int_{\mathbb{R}^{N}}\left(f_{u}^{\prime}\left(x, u_{\mu}\right)-f_{u}^{\prime}(x, 0)\right) v_{n}^{2}=1, \quad \int_{\mathbb{R}^{N}}\left|\nabla v_{n}\right|^{2}+\left(1-f_{u}^{\prime}(x, 0)\right) v_{n}^{2} \longrightarrow \lambda_{1}
$$

and $\left\{v_{n}\right\}$ is bounded in $H^{1}\left(\mathbb{R}^{N}\right)$. Without loss of generality (at least by choosing a subsequence) we can assume, for some $v_{0} \in H^{1}\left(\mathbb{R}^{N}\right)$, that

$$
\begin{array}{lll}
v_{n} \longrightarrow v_{0} & \text { weakly in } H^{1}\left(\mathbb{R}^{N}\right) & \text { as } n \longrightarrow \infty, \\
v_{n} \longrightarrow v_{0} & \text { a.e. in } \mathbb{R}^{N} & \text { as } n \longrightarrow \infty .
\end{array}
$$

Hence

$$
\int_{\mathbb{R}^{N}}\left|\nabla v_{0}\right|^{2}+\left(1-f_{u}^{\prime}(x, 0)\right) v_{0}^{2} \leq \underline{\lim }_{n \rightarrow \infty} \int_{\mathbb{R}^{N}}\left|\nabla v_{0}\right|^{2}+\left(1-f_{u}^{\prime}(x, 0)\right) v_{n}^{2} d x=\lambda_{1} .
$$

To prove that $v_{0}$ achieves $\lambda_{1}$, it suffices to show that

$$
\int_{\mathbb{R}^{N}}\left(f_{u}^{\prime}\left(x, u_{\mu}\right)-f_{u}^{\prime}(x, 0)\right) v_{0}^{2}=1 .
$$

For this purpose, we need some estimates of $f(x, t)$. By $\left.f_{5}\right)$, for any $\varepsilon>0$ we can find $C_{\varepsilon}>0$ such that

$$
\left\{\begin{array}{l}
f_{u}^{\prime \prime}(x, t) \leq \varepsilon t^{-1}+C_{\varepsilon} t^{q-1} \\
f_{u}^{\prime}(x, t) \leq \varepsilon \ln t+\frac{C_{\varepsilon}}{q} t^{q}
\end{array} \quad \text { for } x \in \mathbb{R}^{N}, t>0,\right.
$$

where $0<q<\frac{4}{N-2}$. Consequently, there is a constant $C>0$ such that

$$
f_{u}^{\prime}(x, t) \leq C t^{q} \quad \text { for } x \in \mathbb{R}^{N}, t>1 .
$$


From (3.7) and (3.8) we deduce that for any $\varepsilon>0$, there exists $C_{\varepsilon}>0$ such that

$$
\left|f_{u}^{\prime}(x, t)-f_{u}^{\prime}(x, 0)\right|<\varepsilon+C_{\varepsilon} t^{q} \quad \text { for all } x \in \mathbb{R}^{N}, t>0
$$

For any fixed $R>0$, let $B_{R}=\left\{x \in \mathbb{R}^{N}|| x \mid<R\right\}$. We have

$$
\begin{aligned}
\int_{\mathbb{R}^{N}}\left|f_{u}^{\prime}\left(x, u_{\mu}\right)-f_{u}^{\prime}(x, 0)\right|\left(v_{n}-v_{0}\right)^{2} d x \\
\leq \int_{B_{R}}\left|f_{u}^{\prime}\left(x, u_{\mu}\right)-f_{u}^{\prime}(x, 0)\right|\left|v_{n}-v_{0}\right|^{2} d x \\
\quad+\int_{\mathbb{R}^{N} \backslash B_{R}}\left|f_{u}^{\prime}\left(x, u_{\mu}\right)-f_{u}^{\prime}(x, 0)\right|\left|v_{n}-v_{0}\right|^{2} d x \\
\leq \int_{B_{R}}\left(\varepsilon+C_{\varepsilon} u_{\mu}^{q}\right)\left|v_{n}-v_{0}\right|^{2}+\int_{\mathbb{R}^{N} \backslash B_{R}}\left(\varepsilon+C_{\varepsilon} u_{\mu}^{q}\right)\left|v_{n}-v_{0}\right|^{2} d x \\
\leq \varepsilon \int_{\mathbb{R}^{N}}\left|v_{n}-v_{0}\right|^{2} d x+C_{\varepsilon}\left[\left(\int_{B_{R}} u_{\mu}^{q+2}\right)^{\frac{q}{q+2}}\left(\int_{B_{R}}\left|v_{n}-v_{0}\right|^{q+2}\right)^{\frac{2}{q+2}}\right] \\
\quad+C_{\varepsilon}\left[\left(\int_{\mathbb{R}^{N} \backslash B_{R}} u_{\mu}^{q+2}\right)^{\frac{q}{q+2}}\left(\int_{\mathbb{R}^{N} \backslash B_{R}}\left|v_{n}-v_{0}\right|^{q+2} d x\right)^{\frac{2}{q+2}}\right] .
\end{aligned}
$$

Since $v_{n} \rightarrow v_{0}$ strongly in $L^{s}\left(B_{R}\right)$ for $2 \leq s<\frac{2 N}{N-2},\left\{v_{n}\right\}$ is a bounded sequence in $H^{1}\left(\mathbb{R}^{N}\right)$. Taking $n \rightarrow \infty$, then $R \rightarrow \infty$ and finally $\varepsilon \rightarrow 0^{+}$we deduce (3.6). Therefore, $v_{0}$ achieves $\lambda_{1}$. Clearly $\left|v_{0}\right|$ also achieves $\lambda_{1}$. Hence we may assume $v_{0} \geq 0$ in $\mathbb{R}^{N}$ and $v_{0}$ satisfies

$$
-\triangle v_{0}+\left(1-f_{u}^{\prime}(x, 0)\right) v_{0}=\lambda_{1}\left(f_{u}^{\prime}\left(x, u_{\mu}\right)-f_{u}^{\prime}(x, 0)\right) v_{0} .
$$

Once again, by the maximum principle for weak solutions we deduce that $v_{0}>0$ in $\mathbb{R}^{N}$.

We will now prove that $\lambda_{1}>1$. By the definition of $u_{\mu}$ we obtain for any $\mu_{1}<\mu_{2}$

$$
\begin{aligned}
-\triangle\left(u_{\mu_{2}}-u_{\mu_{1}}\right)+\left(u_{\mu_{2}}-u_{\mu_{1}}\right) & =f\left(x, u_{\mu_{2}}\right)-f\left(x, u_{\mu_{1}}\right)+\left(\mu_{2}-\mu_{1}\right) h(x) \\
& \geq f_{u}^{\prime}\left(x, u_{\mu_{1}}\right)\left(u_{\mu_{2}}-u_{\mu_{1}}\right)+\left(\mu_{2}-\mu_{1}\right) h(x)
\end{aligned}
$$

Multiplying (3.12) by $v_{0}$ and integrating it over $\mathbb{R}^{N}$, we get

$$
\int_{\mathbb{R}^{N}} \nabla\left(u_{\mu_{2}}-u_{\mu_{1}}\right) \nabla v_{0}+\left(u_{\mu_{2}}-u_{\mu_{1}}\right) v_{0} d x>\int_{\mathbb{R}^{N}} f_{u}^{\prime}\left(x, u_{\mu_{1}}\right)\left(u_{\mu_{2}}-u_{\mu_{1}}\right)
$$


By (3.11) we have

$$
\begin{aligned}
& \int_{\mathbb{R}^{N}} \nabla\left(u_{\mu_{2}}-u_{\mu_{1}}\right) \nabla v_{0}+\left(u_{\mu_{2}}-u_{\mu_{1}}\right) v_{0} \\
& \quad=\lambda_{1} \int_{\mathbb{R}^{N}}\left(f_{u}^{\prime}\left(x, u_{\mu_{2}}\right)-f_{u}^{\prime}(x, 0)\right)\left(u_{\mu_{2}}-u_{\mu_{1}}\right) v_{0}+\int_{\mathbb{R}^{N}} f_{u}^{\prime}(x, 0)\left(u_{\mu_{2}}-u_{\mu_{1}}\right) v_{0} .
\end{aligned}
$$

By (3.13) and (3.14) we deduce that

$$
\begin{aligned}
\lambda_{1} \int_{\mathbb{R}^{N}}\left(f_{u}^{\prime}\left(x, u_{\mu_{2}}\right)-f_{u}^{\prime}(x, 0)\right)\left(u_{\mu_{2}}-u_{\mu_{1}}\right) & v_{0} \\
& >\int_{\mathbb{R}^{N}}\left(f_{u}^{\prime}\left(x, u_{\mu_{2}}\right)-f_{u}^{\prime}(x, 0)\right)\left(u_{\mu_{2}}-u_{\mu_{1}}\right) v_{0},
\end{aligned}
$$

which implies that $\lambda_{1}>1$.

By the fact that $\lambda_{1}>1$ we have

$$
\int_{\mathbb{R}^{N}}|\nabla v|^{2}+\left(1-f_{u}^{\prime}(x, 0)\right) v^{2} d x \geq \lambda_{1} \int_{\mathbb{R}^{N}}\left[f_{u}^{\prime}\left(x, u_{\mu}\right)-f_{u}^{\prime}(x, 0)\right) v^{2} d x
$$

for all $v \in H^{1}\left(\mathbb{R}^{N}\right)$.

Lemma 3.4. The problem $(1.1)_{\mu}$ has no solution if $\mu>\mu_{2}$, where $\mu_{2}$ is some positive constant.

Proof. Let $u$ be a positive solution of $(1.1)_{\mu}$ and

$$
w_{\varepsilon}=(N(N-2) \varepsilon)^{\frac{N-2}{4}}\left(\frac{1}{\varepsilon+|x|^{2}}\right)^{\frac{N-2}{2}} .
$$

Then for any $\varepsilon>0$

$$
-\triangle u w_{\varepsilon}^{2^{*}}+u w_{\varepsilon}^{2^{*}}=f(x, u) w_{\varepsilon}^{2^{*}}=f(x, u) w_{\varepsilon}^{2^{*}}+\mu h(x) w_{\varepsilon}^{2^{*}} .
$$

From $\mathrm{f}_{2}$ ) we may conclude that for any $M>0$ there exists a constant $C>0$ such that

$$
f(x, u) \geq M u-C \quad \text { for all } u>0 \text { and } x \in \mathbb{R}^{N} .
$$

It follows from (3.16), (3.17) that

$$
-\int_{\mathbb{R}^{N}} \Delta u w_{\varepsilon}^{2^{*}} d x+\int_{\mathbb{R}^{N}} u w_{\varepsilon}^{2^{*}} d x \geq \int_{\mathbb{R}^{N}}\left((M u-C) w_{\varepsilon}^{2^{*}}+\mu h(x) w_{\varepsilon}^{2^{*}}\right) d x .
$$


Next we claim that

$$
\int_{\mathbb{R}^{N}} \triangle u w_{\varepsilon}^{2^{*}} d x=\int_{\mathbb{R}^{N}} u \triangle w_{\varepsilon}^{2^{*}} d x .
$$

In fact, for any $R>0$, letting $B(R)$ be a ball of radius $R$, we have

$$
\begin{aligned}
\int_{B(R)} & \triangle u w_{\varepsilon}^{2^{*}} d x-\int_{B(R)} u \Delta w_{\varepsilon}^{2^{*}} d x \\
& =\int_{\partial B(R)}\left(\frac{\partial u}{\partial n} w_{\varepsilon}^{2^{*}}-\frac{\partial w_{\varepsilon}^{2^{*}}}{\partial n} u\right) d S \\
& \leq w_{\varepsilon}^{2^{*}}(R) \int_{\partial B(R)}|\nabla u| d S+\left|\nabla w_{\varepsilon}^{2^{*}}\right|(R) \int_{\partial B(R)}|u| d S \\
& =O\left(R^{-2 N}\right) \int_{\partial B(R)}(|\nabla u|+u) d S \\
& \leq O\left(R^{-2 N}\right) R^{\frac{N-1}{2}}\left[\left(\int_{\partial B(R)}|\nabla u|^{2} d S\right)^{\frac{1}{2}}+\left(\int_{\partial B(R)} u^{2} d S\right)^{\frac{1}{2}}\right] \\
& =O\left(R^{-\frac{3 N+1}{2}}\right)\left[\left(\int_{\partial B(R)}|\nabla u|^{2} d S\right)^{\frac{1}{2}}+\left(\int_{\partial B(R)} u^{2} d S\right)^{\frac{1}{2}}\right] .
\end{aligned}
$$

Then from the fact that $u \in H^{1}\left(\mathbb{R}^{N}\right)$ we see that the right-hand side approaches 0 on a sequence of radii $R_{i} \rightarrow \infty$.

From (3.18), (3.19) we get

$$
\begin{array}{rl}
\mu \int_{\mathbb{R}^{N}} & h(x) w_{\varepsilon}^{2^{*}} d x \\
\leq & \leq \int_{\mathbb{R}^{N}} u \triangle w_{\varepsilon}^{2^{*}} d x-M \int_{\mathbb{R}^{N}} w_{\varepsilon}^{2^{*}} u d x+C \int_{\mathbb{R}^{N}} w_{\varepsilon}^{2^{*}} d x+\int_{\mathbb{R}^{N}} w_{\varepsilon}^{2^{*}} u d x \\
\quad & C \int_{\mathbb{R}^{N}} w_{\varepsilon}^{2^{*}} d x+\int_{\mathbb{R}^{N}}\left(1-M-\frac{\triangle w_{\varepsilon}^{2^{*}}}{w_{\varepsilon}^{2^{*}}}\right) w_{\varepsilon}^{2^{*}} u d x .
\end{array}
$$

From the definition of $w_{\varepsilon}$ we get

$$
\begin{aligned}
\frac{\Delta w_{\varepsilon}^{2^{*}}}{w_{\varepsilon}^{2^{*}}} & =\frac{\triangle\left(\varepsilon+|x|^{2}\right)^{-N}}{\left(\varepsilon+|x|^{2}\right)^{-N}}=2 N(N+2)\left(\varepsilon+|x|^{2}\right)^{-2}\left(|x|^{2}-\frac{N}{N+2} \varepsilon\right) \\
& \geq 2 N(N+2)\left(\varepsilon+0^{2}\right)^{-2}\left(0^{2}-\frac{N}{N+2} \varepsilon\right)=-2 N^{2} \varepsilon^{-1} .
\end{aligned}
$$

So (3.20) becomes

$$
\mu \int_{\mathbb{R}^{N}} h(x) w_{\varepsilon}^{2^{*}} d x \leq C \int_{\mathbb{R}^{N}} w_{\varepsilon}^{2^{*}} d x+\left(2 N^{2} \varepsilon^{-1}+1-M\right) \int_{\mathbb{R}^{N}} w_{\varepsilon}^{2^{*}} u d x .
$$


If we choose $M=2 N^{2} \varepsilon^{-1}+1$, then by using the fact that

$$
\int_{\mathbb{R}^{N}}\left|\nabla w_{\varepsilon}\right|^{2} d x=\int_{\mathbb{R}^{N}} w_{\varepsilon}^{\frac{2 N}{N-2}} d x=S^{\frac{N}{2}}
$$

we have

$$
\mu \leq \inf _{\varepsilon>0}\left\{\frac{C_{\varepsilon} \int_{\mathbb{R}^{N}} w_{\varepsilon}^{2^{*}} d x}{\int_{\mathbb{R}^{N}} h(x) w_{\varepsilon}^{2^{*}} d x}\right\}=\inf _{\varepsilon>0}\left\{\frac{C_{\varepsilon} S^{\frac{N}{2}}}{\int_{\mathbb{R}^{N}} h(x) w_{\varepsilon}^{2^{*}} d x}\right\}=\mu_{2} .
$$

If $f(x, u)=u^{p-1}$ we give the expression of $C_{\varepsilon}$. From (3.17), the constant $C$ must satisfy

$$
C \geq M u-u^{2^{*}-1}
$$

Letting $h(u)=M u-f(x, u)$ for $p=2^{*}-1$, it is easy to verify that $u=\left(\frac{M}{p-1}\right)^{\frac{1}{p-2}}$ is the unique critical point which is a maximum of $h(u)$. From $h(0)=0$ and $h(u) \rightarrow-\infty$ as $u \rightarrow+\infty$ we have

$$
\sup _{u \geq 0} h(u)=h\left(\left(\frac{M}{p-1}\right)^{\frac{1}{p-2}}\right) .
$$

So we can take

$$
\begin{aligned}
C=C_{\varepsilon} & =\sup _{u \geq 0} h(u) \\
& =(p-2)\left(\frac{M}{p-1}\right)^{\frac{p-1}{p-2}} \\
& =\frac{4}{N-2}\left(\frac{\left(2 N^{2} \varepsilon^{-1}+1\right)(N-2)}{(N+2)}\right)^{\frac{N+2}{4}} ;
\end{aligned}
$$

then $C$ satisfies (3.21).

From Lemma 3.2 and Lemma 3.4 we have

Corollary 3.5. Suppose $\left.\mathrm{f}_{1}\right), \mathrm{f}_{2}$ ) and $h$ ) Then $\mu^{*}$ is bounded and there is no solution for problem $(1.1)_{\mu}$ if $\mu>\mu^{*}$, where $\mu^{*}$ is given by (3.2).

Proof of Theorem 1.1. From Lemma 3.2 and Corollary 3.5 we only need to prove that problem $(1.1)_{\mu^{*}}$ possesses a unique solution. 
Now we prove that $(1.1)_{\mu}$ has a unique solution if $\mu=\mu^{*}$. Hence for the rest of this section we will assume that $p \in\left(2, \frac{2 N}{N-2}\right]$. We shall use Lemma 3.6-3.7. The proofs of Lemma 3.6 and Lemma 3.7 will be given later. From Lemma 3.3 we have

$$
\int_{\mathbb{R}^{N}}\left(\left|\nabla u_{\mu}\right|^{2}+u_{\mu}^{2}\right) d x-\int_{\mathbb{R}^{N}} f_{u}^{\prime}\left(x, u_{\mu}\right) d x>0
$$

and also we have

$$
\int_{\mathbb{R}^{N}}\left(\left|\nabla u_{\mu}\right|^{2}+u_{\mu}^{2}\right) d x-\int_{\mathbb{R}^{N}} f\left(x, u_{\mu}\right) u_{\mu} d x-\mu \int_{\mathbb{R}^{N}} h(x) u_{\mu} d x=0 .
$$

By using $\mathrm{f}_{3}$ ) we have

$$
\begin{aligned}
\int_{\mathbb{R}^{N}}\left(\left|\nabla u_{\mu}\right|^{2}+u_{\mu}^{2}\right) d x & =\int_{\mathbb{R}^{N}} f\left(x, u_{\mu}\right) u_{\mu} d x+\mu \int_{\mathbb{R}^{N}} h(x) u_{\mu} d x \\
& \leq \alpha \int_{\mathbb{R}^{N}} f_{u}^{\prime}\left(x, u_{\mu}\right) u_{\mu}^{2} d x-\mu \int_{\mathbb{R}^{N}} h(x) u_{\mu} d x \\
& <\frac{1}{\alpha} \int_{\mathbb{R}^{N}}\left(\left|\nabla u_{\mu}\right|^{2}+u_{\mu}^{2}\right) d x+\mu^{*}\|h\|_{H^{-1}}\left\|u_{\mu}\right\|_{H^{1}} \\
& \leq\left(\frac{1}{\alpha}+\frac{\delta \mu^{*}}{2}\right)\left\|u_{\mu}\right\|^{2}+\frac{\mu^{*}}{2 \delta}\|h\|_{H^{-1}}^{2}
\end{aligned}
$$

for any $\delta>0$. Since $\alpha>1$ we can obtain that

$$
\left\|u_{\mu}\right\|_{H^{1}\left(\mathbb{R}^{N}\right)} \leq C<+\infty
$$

for all $\mu \in\left(0, \mu^{*}\right)$ by taking $\delta$ small enough. Since because of (3.3) the solution $u_{\mu}$ is monotone increasing with respect to $\mu$, we may suppose that

$$
u_{\mu} \longrightarrow u_{\mu^{*}} \quad \text { weakly in } H^{1}\left(\mathbb{R}^{N}\right) \text { as } \mu \longrightarrow \mu^{*},
$$

and hence $u_{\mu^{*}}$ is a solution of $(1.1)_{\mu^{*}}$ if $p \in\left(2, \frac{2 N}{N-2}\right]$. The uniqueness of $u_{\mu^{*}}$ is obtained by Lemma 3.7.

Lemma 3.6. Suppose $\left.\mathrm{f}_{1}\right), \mathrm{f}_{2}$ ) with $p \leq \frac{2 N}{N-2}$ if $N \geq 3$. Assume that $u_{\mu}$ is a solution of $(1.1)_{\mu}$ for which $\lambda_{1}>1$. Then for any $g(x) \in H^{-1}\left(\mathbb{R}^{N}\right)$, the problem

$$
-\Delta w+w=f_{u}^{\prime}\left(x, u_{\mu}\right) w+g(x) \quad w \in H^{1}\left(\mathbb{R}^{N}\right)
$$

has a solution (here we suppose $u_{0} \equiv 0$ ), where $\lambda_{1}$ is the first eigenvalue given by (3.4). 
Proof. Consider the functional

$$
\Phi(w)=\frac{1}{2} \int_{\mathbb{R}^{N}}\left(|\nabla w|^{2}+w^{2}\right) d x-\frac{1}{2} \int_{\mathbb{R}^{N}} f_{u}^{\prime}\left(x, u_{\mu}\right) w^{2} d x-\int g(x) w d x,
$$

$w \in H^{1}\left(\mathbb{R}^{N}\right)$. From (3.15), Holder's inequality and Young's inequality we have

$$
\begin{aligned}
\Phi(w)=\frac{1}{2} & \int_{\mathbb{R}^{N}}\left(|\nabla w|^{2}+\left(1-f_{u}^{\prime}(x, 0)\right) w^{2}\right) d x \\
& -\frac{1}{2} \int_{\mathbb{R}^{N}}\left(f_{u}^{\prime}\left(x, u_{\mu}\right)-f_{u}^{\prime}(x, 0)\right) w^{2} d x-\int g(x) w d x \\
\geq \frac{1}{2} & \int_{\mathbb{R}^{N}}\left(|\nabla w|^{2}+\left(1-f_{u}^{\prime}(x, 0)\right) w^{2}\right) d x \\
& -\frac{1}{2 \lambda_{1}} \int_{\mathbb{R}^{N}}\left(|\nabla w|^{2}+\left(1-f_{u}^{\prime}(x, 0)\right) w^{2}\right) d x-\int g(x) w d x \\
\geq & \left(\frac{1}{2}-\frac{1}{2 \lambda_{1}}\right) \int_{\mathbb{R}^{N}}|\nabla w|^{2}+\left(1-f_{u}^{\prime}(x, 0)\right) w^{2} d x-\frac{\varepsilon}{2}\|w\|_{H^{1}}^{2}-\frac{C_{\varepsilon}}{2}\|g\|_{H^{-1}}^{2} .
\end{aligned}
$$

By $\mathrm{f}_{2}$ ) we have $f_{t}^{\prime}(x, 0)=\lim _{t \rightarrow \infty} \frac{|f(x, t)|}{t} \leq \lim _{t \rightarrow 0} C_{1} t^{p-2}+C_{2}=C_{2}$ for all $x \in \mathbb{R}^{N}$ and $1-f_{u}^{\prime}(x, 0) \geq 1-C_{2}>0$ (because $\left.C_{2} \in(0,1)\right)$. Thus

$$
\begin{aligned}
\Phi(w) & \geq\left(\frac{1}{2}-\frac{1}{2 \lambda_{1}}\right) \int_{\mathbb{R}^{N}}|\nabla w|^{2}+\left(1-C_{2}\right) w^{2} d x-\frac{\varepsilon}{2}\|w\|_{H^{1}}^{2}-\frac{C_{\varepsilon}}{2}\|g\|_{H^{-1}}^{2} \\
& \geq\left[\frac{1}{2}\left(1-\frac{1}{\lambda_{1}}\right)\left(1-C_{2}\right)-\frac{\varepsilon}{2}\right]\|w\|_{H^{1}}^{2}-\frac{C_{\varepsilon}}{2}\|g\|_{H^{-1}}^{2} \\
& \geq C\|g\|_{H^{-1}}^{2}
\end{aligned}
$$

if we choose $\varepsilon$ small.

Let $\left\{w_{n}\right\} \subset H^{1}\left(\mathbb{R}^{N}\right)$ be the minimizing sequence of the variational problem

$$
d=\inf \left\{\Phi(w) \mid w \in H^{1}\left(\mathbb{R}^{N}\right)\right\}
$$

From (3.23) we have

$$
\begin{aligned}
{\left[\frac{1}{2}\left(1-\frac{1}{\lambda_{1}}\right)\left(1-C_{2}\right)-\frac{\varepsilon}{2}\right]\left\|w_{n}\right\|_{H^{1}}^{2} } & \leq \Phi\left(w_{n}\right)+\frac{C_{\varepsilon}}{2}\|g\|_{H^{-1}}^{2} \\
& \leq d+\frac{C_{\varepsilon}}{2}\|g\|_{H^{-1}}^{2}+o(1) \quad \text { as } n \longrightarrow \infty
\end{aligned}
$$

By $\lambda_{1}>1$ and $C_{2} \in(0,1)$ we deduce that $\left\{w_{n}\right\}$ is bounded in $H^{1}\left(\mathbb{R}^{N}\right)$ if we choose $\varepsilon$ small. So we may suppose that

$$
\begin{array}{lll}
w_{n} \longrightarrow w & \text { weakly in } H^{1}\left(\mathbb{R}^{N}\right) & \text { as } n \longrightarrow \infty, \\
w_{n} \longrightarrow w & \text { a.e. in } \mathbb{R}^{N} & \text { as } n \longrightarrow \infty .
\end{array}
$$


By Fatou's lemma

$$
\|w\|_{H^{1}\left(\mathbb{R}^{N}\right)}^{2} \leq \underline{\lim }_{n \rightarrow \infty}\left\|w_{n}\right\|_{H^{1}\left(\mathbb{R}^{N}\right)}^{2} .
$$

We now prove that

$$
\int_{\mathbb{R}^{N}}\left(f_{u}^{\prime}\left(x, u_{\mu}\right)-f_{u}^{\prime}(x, 0)\right)\left(w_{n}-w\right)^{2} d x \longrightarrow 0 \quad \text { as } n \longrightarrow \infty .
$$

In fact, by (3.9), for any $\varepsilon>0, R>0$, we have

$$
\begin{aligned}
\int_{\mathbb{R}^{N}}( & \left.f_{u}^{\prime}\left(x, u_{\mu}\right)-f_{u}^{\prime}(x, 0)\right)\left(w_{n}-w\right)^{2} d x \\
\leq & \int_{B_{R}(0)}\left(f_{u}^{\prime}\left(x, u_{\mu}\right)-f_{u}^{\prime}(x, 0)\right)\left(w_{n}-w\right)^{2} d x \\
& \quad+\int_{\mathbb{R}^{N} \backslash B(0)}\left(f_{u}^{\prime}\left(x, u_{\mu}\right)-f_{u}^{\prime}(x, 0)\right)\left(w_{n}-w\right)^{2} d x \\
\leq & \int_{B_{R}(0)}\left(\epsilon+C_{\varepsilon} u_{\mu}^{q}\right)\left(w_{n}-w\right)^{2} d x+\int_{\mathbb{R}^{N} \backslash B_{R}}\left(\varepsilon+C_{\varepsilon} u_{\mu}^{q}\right)\left(w_{n}-w\right)^{2} d x \\
\leq & \varepsilon \int_{\mathbb{R}^{N}}\left|w_{n}+w\right|^{2} d x+C_{\varepsilon}\left[\left(\int_{B_{R}} u_{\mu}^{q+2}\right)^{\frac{q}{q+2}}\left(\int_{B_{R}}\left|w_{n}+w\right|^{q+2}\right)^{\frac{2}{q+2}}\right] \\
& \quad+C_{\varepsilon}\left[\left(\int_{\mathbb{R}^{N} \backslash B_{R}} u_{\mu}^{q+2}\right)^{\frac{q}{q+2}}\left(\int_{\mathbb{R}^{N} \backslash B_{R}}\left|v_{n}-v_{0}\right|^{q+2}\right)^{\frac{2}{q+2}}\right] .
\end{aligned}
$$

Since $w_{n} \rightarrow w$ strongly in $L^{s}\left(B_{R}\right)$ for $2 \leq s<\frac{2 N}{N-2}$, and $\left\{w_{n}\right\}$ is a bounded sequence in $H^{1}\left(\mathbb{R}^{N}\right)$, taking $n \rightarrow \infty$, then $R \rightarrow \infty$, and finally $\varepsilon \rightarrow 0^{+}$, we deduce our claim.

From (3.24) and the definition of weak convergence we can easily deduce that

$$
\int_{\mathbb{R}^{N}}\left(f_{u}^{\prime}\left(x, u_{\mu}\right)-f_{u}^{\prime}(x, 0)\right) w_{n}^{2} d x \longrightarrow \int_{\mathbb{R}^{N}}\left(f_{u}^{\prime}\left(x, u_{\mu}\right)-f_{u}^{\prime}(x, 0)\right) w^{2} d x
$$

and

$$
\int g w_{n} \longrightarrow \int g w
$$

as $n \rightarrow \infty$. Thus

$$
\begin{aligned}
\Phi(w)= & \frac{1}{2} \int|\nabla w|^{2}+w^{2} d x-\frac{1}{2} \int f_{u}^{\prime}\left(x, u_{\mu}\right) w^{2} d x-\int g(x) w d x \\
\leq & \frac{1}{2} \underline{\lim }_{n \rightarrow \infty} \int|\nabla w|^{2}+w^{2} d x-\frac{1}{2} \lim _{n \rightarrow \infty} \int f_{u}^{\prime}\left(x, u_{\mu}\right) w_{n}^{2} d x \\
& \quad-\lim _{n \rightarrow \infty} \int g(x) w_{n} d x \\
= & \varliminf_{n \rightarrow \infty} \Phi\left(w_{n}\right)=d=\operatorname{linf}_{w \in H^{1}} \Phi(w),
\end{aligned}
$$


and hence

$$
\Phi(w)=d,
$$

which gives that $w$ is a solution of $(3.22)_{\mu}$.

Lemma 3.7. Let $p \in\left(2, \frac{2 N}{N-2}\right]$ and let $u_{\mu^{*}}$ be a solution of $(1.1)_{\mu^{*}}$ Then problem $(1.1)_{\mu^{*}}$ has its first eigenvalue $\lambda_{1}\left(\mu^{*}\right)=1$. Moreover, the solution $u_{\mu^{*}}$ is unique.

Proof. Define

$$
F: \mathbb{R} \times H^{1}\left(\mathbb{R}^{N}\right) \longrightarrow H^{-1}\left(\mathbb{R}^{N}\right)
$$

by

$$
F(\mu, u)=\triangle u-u+f\left(x, u^{+}\right)+\mu h(x) .
$$

Since $\lambda_{1}(\mu)>1$ for $\mu \in\left(0, \mu^{*}\right)$ at $u_{\mu}$, it follows that $\lambda_{1}\left(\mu^{*}\right) \geq 1$. If $\lambda_{1}\left(\mu^{*}\right)>1$, the equation $F_{u}\left(\mu^{*}, u_{\mu^{*}}\right) \phi=0$ has no nontrivial solution. From Lemma 3.6, $F$ maps $\mathbb{R} \times H^{1}\left(\mathbb{R}^{N}\right)$ onto $H^{-1}\left(\mathbb{R}^{N}\right)$. Applying the implicit function theorem to $F$ we can find a neighborhood $\left(\mu^{*}-\delta, \mu^{*}+\delta\right)$ of $\mu^{*}$ such that $(1.1)_{\mu}$ possesses a solution $u_{\mu}$ if $\mu \in\left(\mu^{*}-\delta, \mu^{*}+\delta\right)$. This is contradictory to the definition of $\mu^{*}$.

Next, we prove that $u_{\mu^{*}}$ is unique. In fact, if problem $(1.1)_{\mu}$ has another solution, $U_{\mu^{*}} \geq u_{\mu^{*}}$. Set $w=U_{\mu^{*}}-u_{\mu^{*}}$. Then we have

$$
-\triangle w+w=f\left(x, w+u_{\mu^{*}}\right)-f\left(x, u_{\mu^{*}}\right), \quad w>0 \text { in } \mathbb{R}^{N} .
$$

By $\lambda_{1}\left(\mu^{*}\right)=1$ it follows that the problem

$$
-\triangle \phi+\phi=f_{u}^{\prime}\left(x, u_{\mu^{*}}\right) \phi, \quad \phi \in H^{1}\left(\mathbb{R}^{N}\right)
$$

possesses a positive solution $\phi_{1}$.

Multiplying (3.25) by $\phi_{1}$ and (3.26) by $w$, integrating, and subtracting, we deduce that

$$
\begin{aligned}
0 & =\int_{\mathbb{R}^{N}}\left[f\left(x, w+u_{\mu^{*}}\right)-f\left(x, u_{\mu^{*}}\right)-f_{u}^{\prime}\left(x, u_{\mu^{*}}\right) w\right] \phi_{1} d x \\
& =\frac{1}{2} \int_{\mathbb{R}^{N}} f_{u}^{\prime \prime}\left(x, \xi_{\mu}\right) w^{2} \phi_{1} d x,
\end{aligned}
$$

where $\xi \in\left(u_{\mu^{*}}, u_{\mu^{*}}+w\right)$. By the assumption $\left.\mathrm{f}_{4}\right)$ we get that $w \equiv 0$. 
4 THE EXISTENCE OF THE SECOND SOLUTION

\section{The existence of the second solution}

In this section we will prove that problem $(1.1)_{\mu}$ possesses a second positive solution $U_{\mu}>u_{\mu}$. First we will use Lemma 3.4 to prove that the minimal solution $u_{\mu}$ is also a local minimizer of $I(u)$ for $\mu \in\left(0, \mu^{*}\right)$.

Lemma 4.1. If $\left.\left.\mathrm{f}_{3}\right), \mathrm{f}_{4}\right)$ hold and $\mu \in\left(0, \mu^{*}\right), p \in\left(2, \frac{2 N}{N-2}\right)$ if $N \geq 3$, then $u_{\mu}$ is a local minimizer of $I$, that is, there exists an $\varepsilon_{0}>0$ such that

$$
I\left(u_{\mu}+v\right)>I\left(u_{\mu}\right) \quad \text { for all } v \in H^{1}\left(\mathbb{R}^{N}\right),\|v\| \leq \varepsilon_{0} .
$$

In particular, we can find a suitable $\eta>0$ such that

$$
I\left(u_{\mu}+v\right)>I\left(u_{\mu}\right)+\eta \quad \text { for }\|v\|=\varepsilon_{0} .
$$

Proof. For every $v \in H^{1}\left(\mathbb{R}^{N}\right)$, we have, using the fact that $u_{\mu}$ is a solution of $(1.1)_{\mu}$,

$$
\begin{aligned}
I\left(u_{\mu}+v\right)= & \frac{1}{2}\left\|u_{\mu}\right\|^{2}+\frac{1}{2}\|v\|^{2}+\int_{\mathbb{R}^{N}} \nabla u_{\mu} \nabla v+u_{\mu} v d x \\
& \quad-\int_{\mathbb{R}^{N}} F\left(x, u_{\mu}+v\right) d x-\mu \int_{\mathbb{R}^{N}} h(x) u_{\mu} d x-\mu \int_{\mathbb{R}^{N}} h(x) v d x \\
= & I\left(u_{\mu}\right)+\frac{1}{2}\|v\|^{2}-\int_{\mathbb{R}^{N}}\left(F\left(x, u_{\mu}+v\right)-F\left(x, u_{\mu}\right)-f\left(x, u_{\mu}\right) v\right) d x \\
= & I\left(u_{\mu}\right)+\frac{1}{2} \int_{\mathbb{R}^{N}}|\nabla v|^{2}+\left(1-f_{u}^{\prime}(x, 0)\right) v^{2} d x \\
& \quad-\int_{\mathbb{R}^{N}}\left(F\left(x, u_{\mu}+v\right)-F\left(x, u_{\mu}\right)-f\left(x, u_{\mu}\right) v-\frac{1}{2} f_{u}^{\prime}(x, 0) v^{2}\right) d x \\
=I & \left(u_{\mu}\right)+\frac{1}{2}\left(1-\frac{1}{\lambda_{1}}\right) \int_{\mathbb{R}^{N}}|\nabla v|^{2}+\left(1-f_{u}^{\prime}(x, 0)\right) v^{2} d x \\
& \quad+\frac{1}{2 \lambda_{1}} \int_{\mathbb{R}^{N}}|\nabla v|^{2}+\left(1-f_{u}^{\prime}(x, 0)\right) v^{2} d x \\
& \quad-\int_{\mathbb{R}^{N}}\left(F\left(x, u_{\mu}+v\right)-F\left(x, u_{\mu}\right)-f\left(x, u_{\mu}\right) v-\frac{1}{2} f_{u}^{\prime}(x, 0) v^{2}\right) d x,
\end{aligned}
$$

where $\lambda_{1}>1$ is given by Lemma 3.3. From $\left.f_{2}\right)$ and Lemma 3.3 we can deduce $1-f_{u}^{\prime}(x, 0) \geq$ $1-C_{2}>0$ and

$$
\frac{1}{\lambda_{1}} \int_{\mathbb{R}^{N}}|\nabla v|^{2}+\left(1-f_{u}^{\prime}(x, 0)\right) v^{2} d x \geq \int_{\mathbb{R}^{N}}\left(f_{u}^{\prime}\left(x, u_{\mu}\right)-f_{u}^{\prime}(x, 0)\right) v^{2} d x .
$$


Thus

$$
\begin{aligned}
I\left(u_{\mu}+v\right) \geq I\left(u_{\mu}\right)+\frac{\lambda_{1}-1}{2 \lambda_{1}}\left(1-C_{2}\right)\|v\|^{2}+\frac{1}{2} \int_{\mathbb{R}^{N}}\left(f_{u}^{\prime}\left(x, u_{\mu}\right)-f_{u}^{\prime}(x, 0)\right) v^{2} d x \\
\quad-\int_{\mathbb{R}^{N}}\left(F\left(x, u_{\mu}+v\right)-F\left(x, u_{\mu}\right)-f\left(x, u_{\mu}\right) v-\frac{1}{2} f_{u}^{\prime}(x, 0) v^{2}\right) d x \\
=I\left(u_{\mu}\right)+\frac{\lambda_{1}-1}{2 \lambda_{1}}\left(1-C_{2}\right)\|v\|^{2} \\
\quad-\int_{\mathbb{R}^{N}}\left(F\left(x, u_{\mu}+v\right)-F\left(x, u_{\mu}\right)-f\left(x, u_{\mu}\right) v-\frac{1}{2} f_{u}^{\prime}\left(x, u_{\mu}\right) v^{2}\right) d x \\
=I\left(u_{\mu}\right)+\frac{\lambda_{1}-1}{2 \lambda_{1}}\left(1-C_{2}\right)\|v\|^{2} \\
\quad-\int_{\mathbb{R}^{N}} \int_{0}^{v}\left(f\left(x, u_{\mu}+s\right)-f\left(x, u_{\mu}\right)\right) d s d x+\frac{1}{2} \int_{\mathbb{R}^{N}} f_{u}^{\prime}\left(x, u_{\mu}\right) v^{2} d x
\end{aligned}
$$

Noticing that $f_{u}^{\prime \prime}(x, t) \geq 0$ for $x \in \mathbb{R}^{N}, t \geq 0$, we have

$$
f\left(x, u_{\mu}\right)-f\left(x, u_{\mu}-s\right) \leq f\left(x, u_{\mu}+s\right)-f\left(x, u_{\mu}\right) \quad \text { for } x \in \mathbb{R}^{N}, s \geq 0
$$

Therefore,

$$
\begin{aligned}
\int_{\mathbb{R}^{N}} & \int_{0}^{v}\left(f\left(x, u_{\mu}+s\right)-f\left(x, u_{\mu}\right)\right) d s d x \\
& \leq \int_{\mathbb{R}^{N}} \int_{0}^{|v|}\left(f\left(x, u_{\mu}+s\right)-f\left(x, u_{\mu}\right)\right) d s d x
\end{aligned}
$$

Thus

$$
\begin{aligned}
I\left(u_{\mu}+v\right) \geq I\left(u_{\mu}\right)+\frac{\lambda_{1}-1}{2 \lambda_{1}}\left(1-C_{2}\right)\|v\|^{2} \\
\quad-\int_{\mathbb{R}^{N}} \int_{0}^{|v|} \int_{0}^{s}\left(f_{u}^{\prime}\left(x, u_{\mu}+t\right)-f_{u}^{\prime}\left(x, u_{\mu}\right)\right) d t d s d x \\
=I\left(u_{\mu}\right)+\frac{\lambda_{1}-1}{2 \lambda_{1}}\left(1-C_{2}\right)\|v\|^{2}-I_{1} .
\end{aligned}
$$

To estimate $I_{1}$ we consider the following two cases: 
(i) If $0 \leq t \leq u_{\mu}$, then by (3.7), for any $\varepsilon>0$, there exists $C_{\varepsilon}>0$ such that

$$
\begin{aligned}
f_{u}^{\prime}\left(x, u_{\mu}+t\right)-f_{u}^{\prime}\left(x, u_{\mu}\right) & =f_{u}^{\prime \prime}\left(x, u_{\mu}+\theta t\right) t \\
& \leq \varepsilon \frac{t}{u_{\mu}+\theta t}+C_{\varepsilon}\left(u_{\mu}+\theta t\right)^{q-1} t \\
& \leq \begin{cases}\varepsilon+\tilde{C}_{\varepsilon} u_{\mu}^{q-1} t & \text { if } q \geq 1, \\
\varepsilon+\tilde{C}_{\varepsilon} u_{\mu}^{q-\delta} t^{\delta} & \text { for some } 0<\delta<q \text { if } q<1 .\end{cases}
\end{aligned}
$$

(ii) If $t>u_{\mu}$, from the fact that $f_{u}^{\prime \prime}(x, s) \geq 0$ for all $x \in \mathbb{R}^{N}, s>0$, we have

$$
\int_{u_{\mu}}^{u_{\mu}+t} f_{u}^{\prime \prime}(x, s) d s \leq \int_{0}^{2 t} f_{u}^{\prime \prime}(x, s) d s .
$$

By (3.8) and $f_{u}^{\prime}(x, 0) \geq 0$ (since $f_{u}^{\prime}(x, 0)=\lim _{t \rightarrow 0^{+}} \frac{f(x, t)}{t} \geq 0$ ), for any $\varepsilon>0$ there exists $C_{\varepsilon}>0$ such that

$$
\begin{aligned}
f_{u}^{\prime}\left(x, u_{\mu}+t\right)-f_{u}^{\prime}\left(x, u_{\mu}\right) & \leq f_{u}^{\prime}(x, 2 t)-f_{u}^{\prime}(x, 0) \leq f_{u}^{\prime}(x, 2 t) \\
& \leq \varepsilon \ln (2 t)+C_{\varepsilon} t^{q} \\
& \leq \varepsilon+\tilde{C}_{\varepsilon} t^{q} .
\end{aligned}
$$

So, in all cases, for any $\varepsilon>0$, there exists $\tilde{C}_{\varepsilon}>0,0<\delta<q$ if $q<1, \delta=1$ if $q \geq 1$, such that

$$
f_{u}^{\prime}\left(x, u_{\mu}+t\right)-f_{u}^{\prime}\left(x, u_{\mu}\right) \leq \varepsilon+\tilde{C}_{\varepsilon}\left(u_{\mu}^{q-\delta} t^{\delta}+t^{q}\right) .
$$

By the Sobolev's inequality and the Hölder's inequality we have

$$
\begin{aligned}
I_{1} & \leq \varepsilon \int_{\mathbb{R}^{N}} v^{2}+\tilde{C}_{\varepsilon} \int_{\mathbb{R}^{N}} \int_{0}^{|v|} \int_{0}^{s}\left(u_{\mu}^{q-\delta} t^{\delta}+t^{q}\right) d t d s d x \\
& \leq C \varepsilon \int_{\mathbb{R}^{N}}|\nabla v|^{2}+v^{2} d x+\tilde{C}_{e} \int_{\mathbb{R}^{N}} \int_{0}^{|v|}\left(u_{\mu}^{q-\delta} s^{1+\delta}+s^{q+1}\right) d s d x \\
& \leq C \varepsilon\|v\|^{2}+\tilde{C}_{\varepsilon} \int_{\mathbb{R}^{N}} u_{\mu}^{q-\delta}|v|^{2+\delta}+|v|^{q+2} d x \\
& \leq C \varepsilon\|v\|^{2}+\tilde{C}_{\varepsilon}\left(\left\|u_{\mu}\right\|_{q+2}^{q-\delta}\|v\|_{q+2}^{2+\delta}+\|v\|_{q+2}^{q+2}\right) \\
& \leq C \varepsilon\|v\|^{2}+\tilde{C}_{\varepsilon}\left\|u_{\mu}\right\|_{q+2}^{q-\delta}\|v\|^{2+\delta}+\tilde{C}_{\varepsilon}\|v\|^{q+2} .
\end{aligned}
$$


Thus for any $\varepsilon>0$, there exists $C_{\varepsilon}>0$ such that

$$
\begin{aligned}
& I\left(u_{\mu}+v\right) \\
& \quad \geq I\left(u_{\mu}\right)+\left(\frac{\lambda_{1}-1}{2 \lambda_{1}}\left(1-C_{2}\right)-C \varepsilon\right)\|v\|^{2}-C_{\varepsilon}\left\|u_{\mu}\right\|^{q-\delta}\|v\|^{2+\delta}-C_{\varepsilon}\|v\|^{q+2} .
\end{aligned}
$$

Taking $\varepsilon_{0}$ small enough so that $\frac{\lambda_{1}-1}{2 \lambda_{1}}\left(1-C_{2}\right)-C \varepsilon>\frac{\lambda_{1}-1}{4 \lambda_{1}}\left(1-C_{2}\right)$ we have

$$
I\left(u_{\mu}+v\right) \geq I\left(u_{\mu}\right)+\frac{\lambda_{1}-1}{4 \lambda_{1}}\left(1-C_{2}\right)\|v\|^{2} \quad \text { for }\|v\| \leq \varepsilon_{0},
$$

from which we deduce (4.1) and (4.2) for suitable $\eta>0$.

Now we introduce the problem at infinity of $(1.1)_{\mu}$,

$$
\left\{\begin{array}{l}
-\triangle u+u=\bar{f}(u), \quad x \in \mathbb{R}^{N}, \\
u \in H^{1}\left(\mathbb{R}^{N}\right) .
\end{array}\right.
$$

Define

$$
I^{\infty}(u)=\frac{1}{2} \int_{\mathbb{R}^{N}}|\nabla u|^{2}+u^{2} d x-\int_{\mathbb{R}^{N}} \bar{F}(u) d x,
$$

where $\bar{F}(u)=\int_{0}^{u} \bar{f}(s) d s$, and

$$
S^{\infty}=\inf \left\{\left.I^{\infty}(u)\left|u \in H^{1}\left(\mathbb{R}^{N}\right), u \triangleq 0, \int_{\mathbb{R}^{N}}\right| \nabla u\right|^{2}+u^{2} d x=\int_{\mathbb{R}^{N}} \bar{f}(u) u d x\right\} .
$$

It is known [BC], [L1] that there exists a positive solution (ground state) $w_{0}$ of (4.9) such that $S^{\infty}=I^{\infty}\left(w_{0}\right)$. It is easy to verify that

$$
\sup _{t \geq 0} I^{\infty}\left(t w_{0}\right)=S^{\infty}
$$

Lemma 4.2. If $\left.\left.\left.\mathrm{f}_{1}\right)-\mathrm{f}_{4}\right), \mathrm{f}_{6}\right)$ hold and $u_{\mu}$ is the minimal solution of $(1.1)_{\mu}$, then there exists $t_{0}>0$ such that

i) $I\left(u_{\mu}+t w_{0}\right)<I\left(u_{\mu}\right)$ for all $t \geq t_{0}$,

ii) $\sup _{t \geq 0} I\left(u_{\mu}+t w_{0}\right)<I\left(u_{\mu}\right)+S^{\infty}$. 
Proof. By the definition of $I(u)$, we have

$$
\begin{aligned}
& I\left(u_{\mu}+t w_{0}\right) \\
& =\frac{1}{2} \int_{\mathbb{R}^{N}}\left|\nabla u_{\mu}\right|^{2}+\left|u_{\mu}\right|^{2} d x+\frac{t^{2}}{2} \int_{\mathbb{R}^{N}}\left|\nabla w_{0}\right|^{2}+w_{0}^{2} d x+t \int_{\mathbb{R}^{N}} \nabla u_{\mu} \nabla w_{0}+u_{\mu} w_{0} d x \\
& \quad \quad-\int_{\mathbb{R}^{N}} F\left(x, u_{\mu}+t w_{0}\right) d x-\mu \int_{\mathbb{R}^{N}} h u_{\mu}-\mu t \int_{\mathbb{R}^{N}} h w_{0} d x \\
& =I\left(u_{\mu}\right)+I^{\infty}\left(t w_{0}\right) \\
& -\int_{\mathbb{R}^{N}} F\left(x, u_{\mu}+t w_{0}\right)-F\left(x, u_{\mu}\right)-\bar{F}\left(t w_{0}\right)-t f\left(x, u_{\mu}\right) w_{0} d x \\
& \leq I\left(u_{\mu}\right)+I^{\infty}\left(t w_{0}\right)-\int_{\mathbb{R}^{N}} \int_{0}^{t w_{0}}\left(f\left(x, u_{\mu}+s\right)-f(x, s)-f\left(x, u_{\mu}\right)\right) d s d x,
\end{aligned}
$$

because $\mathrm{f}_{6}$ ) and $u_{\mu}$ is a solution of $(1.1)_{\mu}$. From $\left.\mathrm{f}_{3}\right)$ and $\mathrm{f}_{4}$ ), we have

$$
\left\{\begin{array}{l}
f\left(x, t_{1}+t_{2}\right) \geq f\left(x, t_{1}\right)+f\left(x, t_{2}\right) \\
f\left(x, t_{1}+t_{2}\right) \not \equiv f\left(x, t_{1}\right)+f\left(x, t_{2}\right)
\end{array}\right.
$$

for all $x \in \mathbb{R}^{N}, t_{1}, t_{2} \geq 0$. By applying (4.14) to (4.13) we obtain

$$
I\left(u_{\mu}+t w_{0}\right) \leq I\left(u_{\mu}\right)+I^{\infty}\left(t w_{0}\right) .
$$

By $\left.\mathrm{f}_{6}\right), I^{\infty}\left(t w_{0}\right) \rightarrow-\infty$ as $t \rightarrow+\infty$ and consequently i) holds.

From i) we know that

$$
\sup _{t \geq 0} I\left(u_{\mu}+t w_{0}\right) \leq \sup _{t \leq t_{0}} I\left(u_{\mu}+t w_{0}\right)
$$

for some $t_{0}>0$. By the continuity of $I\left(u_{\mu}+t w_{0}\right)$ as a function of $t \geq 0$, we can find some $t_{1} \in\left(0, t_{0}\right)$ such that

$$
\sup _{0 \leq t \leq t_{1}} I\left(u_{\mu}+t w_{0}\right)<I\left(u_{\mu}\right)+S^{\infty} .
$$

Thus, to prove ii), we only need to show that

$$
\sup _{t_{1} \leq t \leq t_{0}} I\left(u_{\mu}+t w_{0}\right)<I\left(u_{\mu}\right)+S^{\infty} .
$$


To this end, let us go back to (4.13). We have

$$
\begin{aligned}
& \sup _{t_{1} \leq t \leq t_{0}} I\left(u_{\mu}+t w_{0}\right) \\
& \quad \leq I\left(u_{\mu}\right)+S^{\infty}-\inf _{t_{1} \leq t \leq t_{0}} \int_{\mathbb{R}^{N}} \int_{0}^{t w_{0}}\left(f\left(x, u_{\mu}+s\right)-f(x, s)-f\left(x, u_{\mu}\right)\right) d s d x \\
& \quad<I\left(u_{\mu}\right)+S^{\infty} .
\end{aligned}
$$

Therefore ii) holds.

We say that $\left\{u_{n}\right\} \subset H^{1}\left(\mathbb{R}^{N}\right)$ is a (PS) sequence if $I\left(u_{n}\right)$ is bounded and

$$
I^{\prime}\left(u_{n}\right) \longrightarrow 0 \quad \text { in } H^{-1}\left(\mathbb{R}^{N}\right) \text { as } n \longrightarrow \infty \text {. }
$$

The following theorem provides a precise description for the (PS) sequence of $I$.

Theorem B. Assume that $\left.\left.\mathrm{f}_{1}\right)-\mathrm{f}_{6}\right)$ hold, and $\left\{u_{n}\right\}$ is a $(P S)$ sequence for $I$. Then there is a subsequence of $\left\{u_{n}\right\}$ (still denoted by $\left\{u_{n}\right\}$ ) such that there exist an integer $m \geq 0$, sequences $\left\{x_{n}^{i}\right\} \subset \mathbb{R}^{N}$ for $1 \leq i \leq m$, a solution $U_{\mu}$ of $(1.1)_{\mu}$ and solutions $u^{i}(1 \leq i \leq m)$ of (4.9) satisfying

$$
\begin{aligned}
& u_{n} \longrightarrow U_{\mu} \quad \text { weakly in } H^{1}\left(\mathbb{R}^{N}\right) \text { as } n \longrightarrow \infty, \\
& I\left(u_{n}\right) \longrightarrow I\left(U_{\mu}\right)+\sum_{i=1}^{m} I^{\infty}\left(u^{i}\right), \\
& \left\|u_{n}-\left(U_{\mu}+\sum_{i=1}^{m} u^{i}\left(x-x_{n}^{i}\right)\right)\right\| \longrightarrow 0 \quad \text { as } n \longrightarrow \infty, \\
& \left|x_{n}^{i}\right| \longrightarrow+\infty,\left|x_{n}^{i}-x_{n}^{j}\right| \longrightarrow \infty \quad \text { for } 1 \leq i \neq j \leq m \text { as } n \longrightarrow \infty,
\end{aligned}
$$

where we agree that in the case $m=0$, the above holds without $u^{i},\left\{x_{n}^{i}\right\}$.

Proof. This result can be proved by the arguments in [ZC] (see also, for example, [L1], $[\mathrm{BC}])$.

Proof of Theorem 1.2. We will use the mountain pass lemma without the (PS) condition in $[\mathrm{BN}]$ to obtain the existence of the second positive solution. For this purpose, fix $t_{0}$ 
large enough so that i) in Lemma 4.2 holds for $\left\|t_{0} w_{0}\right\|>\varepsilon_{0}$, where $\varepsilon_{0}$ is chosen as in Lemma 4.1. Let $u_{\mu}$ be the minimal solution. Set

$$
\begin{aligned}
& \Gamma=\left\{\nu \in C\left([0,1], H^{1}\left(\mathbb{R}^{N}\right)\right) \mid \nu(0)=u_{\mu}, \nu(1)=t_{0} w_{0}+u_{\mu}\right\}, \\
& c=\inf _{\nu \in \Gamma} \sup _{s \in[0,1]} I(\nu(s)) .
\end{aligned}
$$

It follows from Lemma 4.1 and Lemma 4.2 that

$$
\eta+I\left(u_{\mu}\right)<c<I\left(u_{\mu}\right)+S^{\infty} .
$$

From the conclusions of Lemma 4.1 and Lemma 4.2, using the mountain pass lemma in $[\mathrm{BN}]$ we can obtain a sequence $\left\{u_{n}\right\} \subset H^{1}\left(\mathbb{R}^{N}\right)$ such that

$$
\begin{array}{ll}
I\left(u_{n}\right) \longrightarrow c & \text { as } n \longrightarrow \infty, \\
I^{\prime}\left(u_{n}\right) \longrightarrow 0 & \text { in } H^{-1}\left(\mathbb{R}^{N}\right) \text { as } n \longrightarrow \infty
\end{array}
$$

Thus, by Theorem B, there exist a subsequence (still denoted by $\left\{u_{n}\right\}$ ), an integer $m \geq 0$, sequences $\left\{x_{n}^{i}\right\}$ in $\mathbb{R}^{N}(1 \leq i \leq m)$, and a solution $U_{\mu}$ of $(1.1)_{\mu}$ and solutions $u^{i}(1 \leq i \leq$ $m$ ) of (4.9) (if $m \geq 1$ ) such that

$$
\begin{aligned}
& c=\lim _{n \rightarrow \infty} I\left(u_{n}\right)=I\left(U_{\mu}\right)+\sum_{i=1}^{m} I^{\infty}\left(u^{i}\right), \\
& \| \begin{array}{l}
u_{n}-U_{\mu}-\sum_{i=1}^{m} u^{i}\left(x-x_{n}^{i}\right) \| \longrightarrow 0 \quad \text { as } n \longrightarrow \infty .
\end{array}
\end{aligned}
$$

We will show that $U_{\mu}$ is a solution of $(1.1)_{\mu}$ different from the minimal solution $u_{\mu}$. Since $u_{\mu}$ is minimal, $U_{\mu}>u_{\mu}$ everywhere or $U_{\mu} \equiv u_{\mu}$. Indeed, if $U_{\mu} \equiv u_{\mu}$ then either $c \geq I\left(u_{\mu}\right)+S^{\infty}(m \geq 1)$ or $c=I\left(u_{\mu}\right)(m=0)$. Both cases contradict $I\left(u_{\mu}\right)<c<$ $I\left(u_{\mu}\right)+S^{\infty}$.

Let $u^{-}=\min \{0, u\}$. From the fact that $U_{\mu}$ solves $(1.1)_{\mu}$ and Remark 1.4

$$
\int_{\mathbb{R}^{N}} f\left(x, U_{\mu}\right) U_{\mu}^{-} d x=0 .
$$

We obtain

$$
\int_{\mathbb{R}^{N}}\left|\nabla U_{\mu}^{-}\right|^{2}+\left|U_{\mu}^{-}\right|^{2}=\int_{\mathbb{R}^{N}} h(x) U_{\mu}^{-} \leq 0
$$

Thus $U_{\mu} \geq 0$. By the maximum principle for weak solutions we have $U_{\mu}>0$ in $\mathbb{R}^{N}$. Hence we have completed the proof of Theorem 1.2. 
REFERENCES

\section{References}

[A] H. Amann, Fixed point equations and nonlinear eigenvalue problems in ordered Banach spaces, SIAM Review 18 (1976) pp. 620-709.

[Ba] A. Bahri, "Critical points at infinity in some variational problems". Pitman Research Notes in Mathematics Series, 182. Longman Scientific and Technical, Harlow; copublished in the United States with John Wiley and Sons, Inc., New York (1989).

[BaLi] A. Bahri and YanYan Li, On a min-max procedure for the existence of a positive solution for certain scalar field equations in $R^{N}$, it Rev. Mat. Iberoamericana 6 (1990) pp. 1-15.

[BaL] A. Bahri and P.-L. Lions, On the existence of a positive solution of semilinear elliptic equations in unbounded domains, Ann. Inst. H. Poincar Anal. Non Linaire 14 (1997) pp. 365-413.

[BC] V. Benci and G. Cerami, Positive solutions of some nonlinear elliptic problems in exterior domains, Arch. Rat'l. Mech. Anal. 99 (1987) pp. 283-300.

[BL] H. Berestycki and P.-L. Lions, Nonlinear scalar field equations, I) Existence of a ground state; II) Existence of infinitely many solutions, Arch. Rat'l. Mech. Anal. 82 (1983) pp. 313-375.

[BN] H. Brezis and L. Nirenberg, Positive solutions of nonlinear elliptic equations involving critical Sobolev exponents, Comm. Pure. Appl. Math. 36 (1983) pp. 437477.

[CR] M.G. Crandall and P.H. Rabinowitz, Bifurcation, perturbation of simple eigenvalues and linearized stability, Arch. Rat'l Mech. Anal. 52 (1973) pp. 161-180.

[CZ] D. M. Cao and H. S. Zhou, Multiple positive solution of nonhomogeneous semilinear elliptic equations in $\mathbb{R}^{N}$, Proc. Royal Society Edinburgh 126A (1996) pp. 443-463. 
REFERENCES

[DL1] Y.B. Deng and Y. Li, Existence and Bifurcation of the Positive Solutions For a Semilinear Equation With Critical Exponent, it J. Diff. Equns. 130(1) (1996) pp. 179-200.

[DL2] Y.B. Deng and Y. Li, Existence and of Multiple Positive Solutions For a Semilinear Elliptic Equation Advances Diff. Equns. 2(3) (1997) pp. 361-382.

[DN] W.-Y. Ding and W.-M. Ni, On the existence of positive entire solution of a semilinear elliptic equation, Arch. Rat'l Mech. Anal. 91 (1986) pp. 283-308.

[E] Lawrence C. Evans, Partial Differential Equations.

[GE] J. Graham-Eagle, Monotone method for semilinear elliptic equations in unbounded domains, J. Math. Anal. Appl. 137 (1989) pp. 122-31.

[GNN] Gidas, Weimin Ni and L. Nirenberg, Symmetry of the positive solutions of nonlinear elliptic equations in $\mathbb{R}^{N}$, Advances in Math. supplementary studies 7A (1981) pp. 369-402.

[G] C.-F. Gui, Existence of multi-bump solutions for nonlinear Schrdinger equations via variational method, Comm. Partial Differential Equations 21, 787-820 (1996).

[KZ] M.-K. Kwong and L.-Q. Zhang, Uniqueness of positive solutions of $\triangle u+f(u)=0$ in an annulus, Diff. Intg'l. Equa. 4 (1991) pp. 583-599.

[L] Y. Li, Remarks on a semilinear elliptic equation on $\mathbb{R}^{N}, J$. Differential Equations 74, 34-49 (1988).

[Li] YanYan Li, Nonautonomous nonlinear scalar field equations, Indiana Univ. Math. J. 39, 283-301 (1990).

[L1] P. L. Lions, The concentration-compactness principle in the calculus of variations, The local compact, part 1 and part 2 Ann.Inst. H.Poincare Anal. Non. Lineaire 1 (1984) pp. 45-109. 
[L2] P.-L. Lions, "On positive solutions of semilinear elliptic equations in unbounded domains". In Nonlinear Diffusion Equation and Their Equilibrium states, W-M. Ni, L. A. Peletier and J. Serrin Eds New York / Berlin: Springer-verlag (1989).

[N] W.-M. Ni, "Some aspects of semilinear elliptic equations on $\mathbb{R}^{N}$. In Nonlinear diffusion equations and their equilibrium states, II (Berkeley, CA, 1986), 171-205, Math. Sci. Res. Inst. Publ., 13, Springer, New York-Berlin (1988).

[PW] X.-B. Pan and X.-F. Wang, Blow-up behavior of ground states of semilinear elliptic equations $R^{n}$ involving critical Sobolev exponents, J. Differential Equations 99 (1992) pp. 78-107.

[W] X.-F. Wang, On location of blow-up of ground states of semilinear elliptic equations in $R^{n}$ involving critical Sobolev exponents, J. Differential Equations 127 (1996) pp. 148-173.

[Y] J.-F.Yang, Positive solution of semilinear elliptic problems in exterior domains, $J$. Diff. Equa. 106 (1993) pp.40-69.

[Z] X. P. Zhu, A perturbation result on positive entire solutions of semilinear elliptic equation, J. Diff. Equns. 92 (1991), 163-78.

[ZC] X. P. Zhu and D. M. Cao, The concentration-compactness principle nonlinear elliptic equations, Acta Math.Sci. 9 (1989) 307-28.

[ZZ] X.P. Zhu and H.S. Zhou, unspecified work.X.-P. Zhu and H.-S. Zhou, Existence of multiple positive solutions of inhomogeneous semilinear elliptic problem in unbounded domains, Proceeding of the Royal Society of Edinburgh 115A (1990) pp. 301-318 . 